\title{
基于分子内交叉氧化偶联反应来非对映选择性构建全碳季碳中心
}

\author{
陈 伟 $a$ 郭人予 $a$ 龚建贤*,a 杨 震*,a,b \\ ( ${ }^{a}$ 北京大学深圳研究生院 省部共建肿瘤化学基因组学国家重点实验室 深圳 518055) \\ $\left({ }^{b}\right.$ 北京大学 北京分子科学国家重点实验室 生物有机分子工程教育部重点实验室 北京 100871)
}

\begin{abstract}
摘要 立体位阻拥挤 $\mathrm{C}-\mathrm{C}$ 键, 尤其是包含全碳季碳中心 $\mathrm{C}-\mathrm{C}$ 键的构建一直是现代有机合成化学的挑战. 利用硝酸铈 铵(CAN)诱导的双烯醇硅醚化合物分子内交叉氧化偶联反应来非对映选择性地构建连续全碳季碳中心以及叔碳中心. 该方法在相对温和的反应条件下, 以 CAN 作为单电子氧化剂, 高产率、高非对映选择性地构建立体位阻拥挤的 C-C 键. 本研究提供了一种通过分子内交叉氧化偶联反应来实现两个不同片段高效连接的有效手段, 可以应用到复杂天然 产物的全合成研究中去.
\end{abstract}

关键词 氧化偶联反应; 季碳中心; 叔碳中心; 方法学

\section{Diastereoselective Construction of All-Carbon Quaternary Stereocenters via Intramolecular Oxidative Cross-Coupling Reaction}

\author{
Chen, $\mathrm{Wei}^{a} \quad$ Guo, Renyu ${ }^{a} \quad$ Gong, Jianxian*,a $\quad$ Yang, Zhen ${ }^{*, a, b}$ \\ ( ${ }^{a}$ State Key Laboratory of Chemical Oncogenomics, Peking University Shenzhen Graduate School, Shenzhen 518055) \\ ( ${ }^{b}$ Key Laboratory of Bioorganic Chemistry and Molecular Engineering of Ministry of Education, Beijing National \\ Laboratory for Molecular Science (BNLMS), Peking University, Beijing 100871)
}

\begin{abstract}
The formation of sterically hindered $\mathrm{C}-\mathrm{C}$ bond represents a great challenge in modern synthetic organic chemistry. A particularly challenging issue is the construction of all-carbon quaternary stereocenters. Herein, a ceric ammonium nitrate (CAN)-mediated intramolecular oxidative cross-coupling of silyl ethers for direct construction of valuable polycyclic scaffolds is described. The reaction enables sterically congested vicinal all-carbon quaternary and tertiary stereocenters to be installed diastereoselectively. The developed method provides a concise and efficient approach for ligation of two different segments through a compact $\mathrm{C}-\mathrm{C}$ bond formation, which has potential applications in the synthesis of complex molecules as well as sterically congested natural products.

Keywords oxidative coupling reaction; quaternary stereocenter; tertiary stereocenter; methodology
\end{abstract}

烯醇化合物的氧化偶联反应作为一种高效构建 CC 键的方法, 可以一步构建 1,4-二羰基化合物, 是有机 合成化学领域研究关注的重点之一 ${ }^{[1]}$. 该类反应的历史 可以追溯到 20 世纪 30 年代. 1935 年, Ivanoff 和 Spassoff 小组 ${ }^{[2]}$ 报道了首例同源氧化偶联反应, 并逐渐发展成为 直接构建 $\mathrm{C}-\mathrm{C}$ 键的有效方法 ${ }^{[3,4]}$. 从合成经济性角度来
看, 由于该反应无需预先对参与反应的碳原子进行官能 团化，显然更加符合 “步骤经济性” ${ }^{[5]}$; 然而，目前该类 型反应最大的挑战在于如何实现立体选择性的交叉氧 化偶联反应. 究其原因在于不同烯醇化合物之间的氧化 偶联反应往往存在较多的竞争反应，如同源二聚反应、 Claisen 缩合反应等，从而限制了其在有机合成中的广

\footnotetext{
* Corresponding authors. E-mail: zyang@pku.edu.cn; gongjx@pku.edu.cn

Received May 10, 2018; revised September 13, 2018; published online September 26, 2018.

Dedicated to Professor Qingyun Chen on the occasion of his 90th birthday.

Project supported by the National Natural Science Foundation of China (Nos. 21772008, 21632002), the Shenzhen Science and Technology Project Program (No. GRCK2017042414425972), the Natural Science Foundation of Guangdong Province (No. 2016A030306011) and the Qingdao National Laboratory for Marine Science and Technology (No. LMDBKF201703).

国家自然科学基金(Nos. 21772008, 21632002)、深圳市科技计划(No. GRCK2017042414425972)、广东省自然科学基金(No. 2016A030306011)和青岛海 洋科学与技术国家实验室开放基金(No. LMDBKF201703)资助项目.
} 
泛应用. 为了实现烯醇化合物的交叉氧化偶联反应，通 常可采取以下三种策略: (1)使用过量的一分子烯醇化合 物 ${ }^{[3 b, 3 c, 6] ;}$ (2)利用两种不同烯醇底物 (如烯胺和烯醇硅醚) 氧化还原电势的差异 ${ }^{[3 \mathrm{~g}, 3 \mathrm{j}, 7]}$; (3) 通过利用双烯醇硅醚的 方式将分子间的反应变成分子内的反应 ${ }^{[8,9]}$. 其中, 利用 双烯醇硅醚底物进行氧化偶联反应时通常能实现较好 的立体选择性, 这使得该方法成为近些年来该领域研究 的热点之一. 1998 年, Schmittel 小组 ${ }^{[8]}$ 报道了首例利用 双烯醇硅醚化合物实现的非对映选择性 $\mathrm{C}-\mathrm{C}$ 键的构建 (Scheme 1). 双烯醇硅醚底物 $\mathbf{1}$ 在硝酸铈铵(CAN)作氧 化剂以及碳酸氢钠作碱的条件下, 能以 $59 \%$ 的产率和 9: 1 的非对映选择性得到偶联产物 $\mathbf{2}$, 反应十分高效. 近年来, Thomson 小组 ${ }^{[4 \mathrm{e}, 4 \mathrm{f}, 4 \mathrm{~h}]}$ 在这一领域做了大量卓有 成效的研究工作, 并取得了一系列研究成果. 2008 年, 该课题组报道了环状双烯醇硅醚底物 $\mathbf{3}$ 在使用与前人报 道的类似反应条件下 ${ }^{[8 a]}$, 可以实现环状底物的交叉氧化 偶联反应，从而非对映选择性地构建化合物 $\mathbf{4}$ 中的 CC 键 (Scheme 1) ${ }^{[9 b]}$. 此外, 该研究小组还利用该反应实 现了单个季碳中心的构建 (Scheme 1$)^{[9 a]}$.

- Schmittel's intramolecular oxidative coupling of acyclic silyl bis-enol ethers (1998)
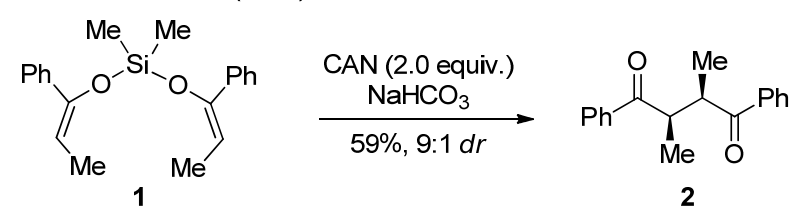

- Thomson's intramolecular oxidative coupling of cyclic silyl bis-enol ethers (2008)<smiles>C=C(C)[C@@H]1C=C(O[Si](OC2=CCCCC2)(C(C)C)C(C)C)C(C)=CC1</smiles>

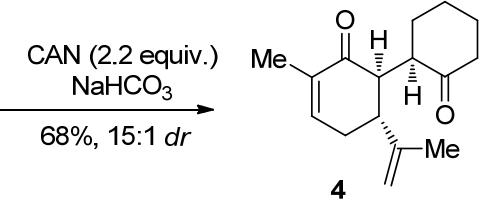<smiles>[R]C(=C)O[Si](C)(C)OC1=C(C)CCc2ccccc21</smiles>
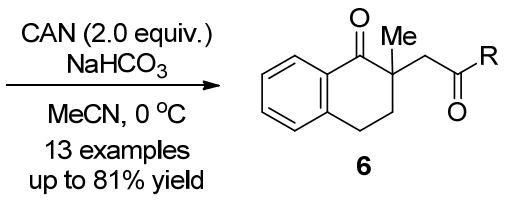

图式 1 已报道的双烯醇硅醚化合物氧化偶联反应

Scheme 1 Oxidative coupling reaction of bis-enol ethers

全碳季碳立体中心广泛存在于多种活性天然产物 分子和功能有机分子中 ${ }^{[10]}$, 正是因为全碳季碳中心独 特的骨架单元，使得天然产物具有三维空间结构，从而 能有效地结合和调控人类疾病的生物靶点 ${ }^{[11]}$. 图 1 展示 了部分具有重要生理活性的代表性天然产物的分子结 构, 包括 nimbin $(7)^{[12]}$, tyrinnal (8) ${ }^{[13]}$, aplyviolene $(\mathbf{9})^{[14]}$.
从结构上看, 它们均包含全碳季碳立体中心且位于分子 左右两个不同片段的连接处. 因此, 如何高效快速构建 该季碳立体中心是实现这些天然产物全合成的关键. 虽 然目前有机化学家已经发展出一系列用于构建全碳季 碳中心的方法，并应用到众多经典天然产物的全合成研 究工作中 ${ }^{[10]}$, 但非对映选择性地构建连续全碳季碳和 叔碳中心结构依然是当今有机合成领域研究的热点和 难点之一.

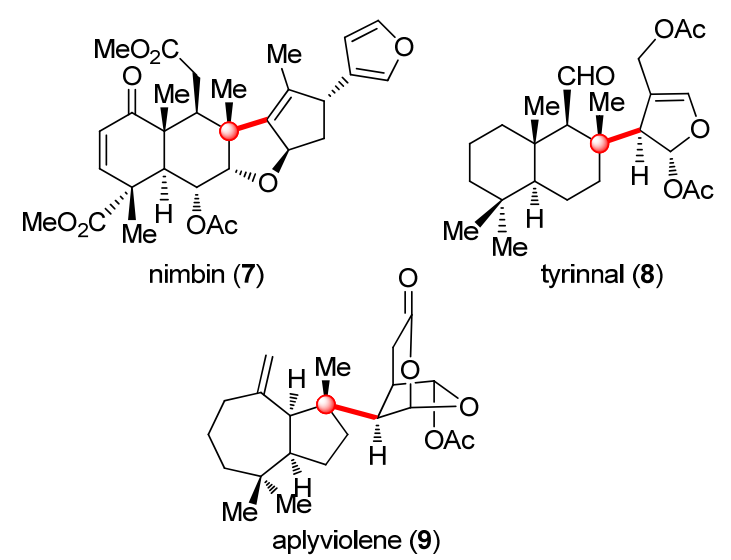

图 1 部分包含全碳季碳立体中心的代表性天然产物 Figure 1 Representative natural products with all-carbon quaternary stereocenters

我们课题组 ${ }^{[15]}$ 一直致力于发展新型高效的有机合 成方法学以实现立体选择性地构建全碳季碳中心, 并将 其应用于复杂天然产物的全合成中. 基于前人报道的工 作以及本课题组的研究基础, 我们发现, 利用四氢荎酮 底物 10 与 $(R)$-或者 $(S)$-香芹酮(carvone) 可以在类似的反 应条件下生成相应的双烯醇硅醚化合物，然后通过 CAN 诱导的分子内交叉氧化偶联反应来实现非对映选 择性地构建偶联产物 11 与 12 中连续全碳季碳以及叔碳 中心(Scheme 2).

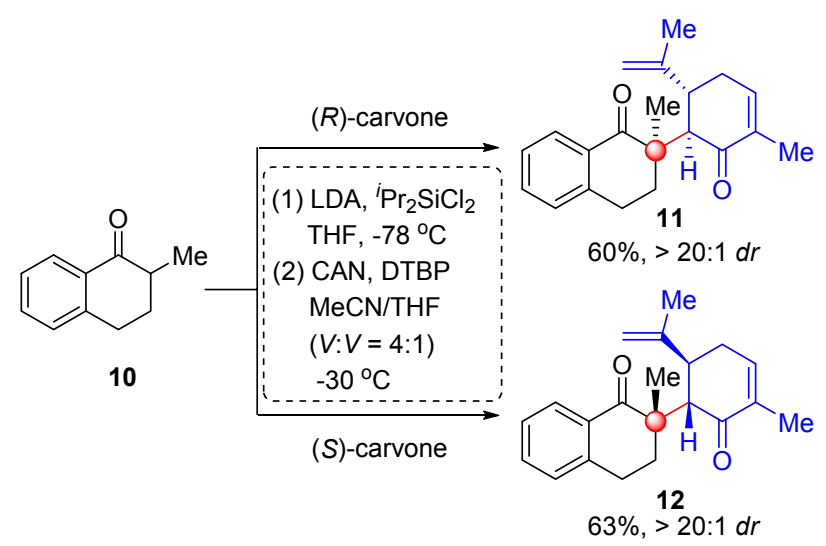

图式 2 双烯醇硅醚化合物分子内交叉氧化偶联反应 Scheme 2 Intramolecular oxidative coupling reaction of bisenol ethers 


\section{1 结果与讨论}

首先，对反应前体 14 进行了制备，如 Scheme 3 所 示. 根据 Thomson 小组 ${ }^{[9]}$ 报道的合成方法进行了部分操 作的改良：首先将四氢荎酮 10 溶解在四氢呋喃(THF) 中, 在二异丙基氨基锂(LDA)作碱的条件下形成烯醇负 离子, 随后与二异丙基二氯化硅反应生成中间体 $\mathbf{A}$; 紧 接着 $\mathbf{A}$ 与原位生成的另一烯醇负离子 $\mathbf{B}$ 反应，以大于 $85 \%$ 的收率(通过 ${ }^{1} \mathrm{H}$ NMR 确定)得到双烯醇硅醚粗产物 14. 需要说明的是, 由于部分双烯醇硅醚底物不稳定, 在快速柱色谱分离时需使用中性或者碱性氧化铝作为 填充剂, 否则底物会降解. 此外, 绝大部分的双烯醇硅 醚底物无需进一步柱色谱分离, 而是经过砂芯漏斗过滤 后旋干溶剂直接用于下一步反应.

在得到足够量的反应底物 14 后, 我们开始对该反 应的条件进行优化, 包括碱、温度、氧化剂、溶剂等因 素，以期得到反应的最优条件(表 1).

首先, 我们以 $\mathrm{CAN}$ 作为单电子氧化剂, 乙腈作为
溶剂在 $-10{ }^{\circ} \mathrm{C}$ 的条件下进行该反应，令人惊喜的是， 反应能以 $45 \%$ 的产率得到单一的 $(>20: 1 d r)$ 偶联产物 11(表 1, Entry 1), 反应产率偏低的原因可能是 CAN 具 有弱酸性从而导致底物发生部分的降解. 因此, 紧接着 对不同的碱进行了探究(Entries 2 5). 可以看出, 不论 是在无机碱还是在有机碱存在的情况下，反应均能提供 偶联产物 11; 其中, 当使用 2,6-二叔丁基吡啶(DTBP)作 碱时能得到最好的产率 $(55 \%$, Entry 5$)$. 随后，还考察不 同单电子氧化剂，包括 $\mathrm{FeCl}_{3}{ }^{[16]}, \mathrm{Mn}(\mathrm{OAc})_{3} \cdot 2 \mathrm{H}_{2} \mathrm{O}^{[17]}$, $\mathrm{Cu}(\mathrm{OAc})_{2}{ }^{[3 \mathrm{~b}]}$ 和 $\mathrm{Cu}(\mathrm{OTf})_{2}{ }^{[18]}$ 对该反应的影响(Entries 6 9), 但并未能进一步提高反应的产率; 而进一步降低反 应温度能稍微提高该反应的产率(Entries 10,11). 最后, 我们得到了该反应的最优条件(Entry 12): 以 CAN 作单 电子氧化剂, DTBP 作碱, 以 $\mathrm{MeCN} / \mathrm{THF}(V: V=4: 1)$ 作溶剂，在一 $30{ }^{\circ} \mathrm{C}$ 反应 $15 \mathrm{~min}$, 能以 $60 \%$ 的产率得到 单一的目标偶联产物 11, 并通过二维核奥弗豪泽增强 谱(NOESY)确定其相对立体化学.

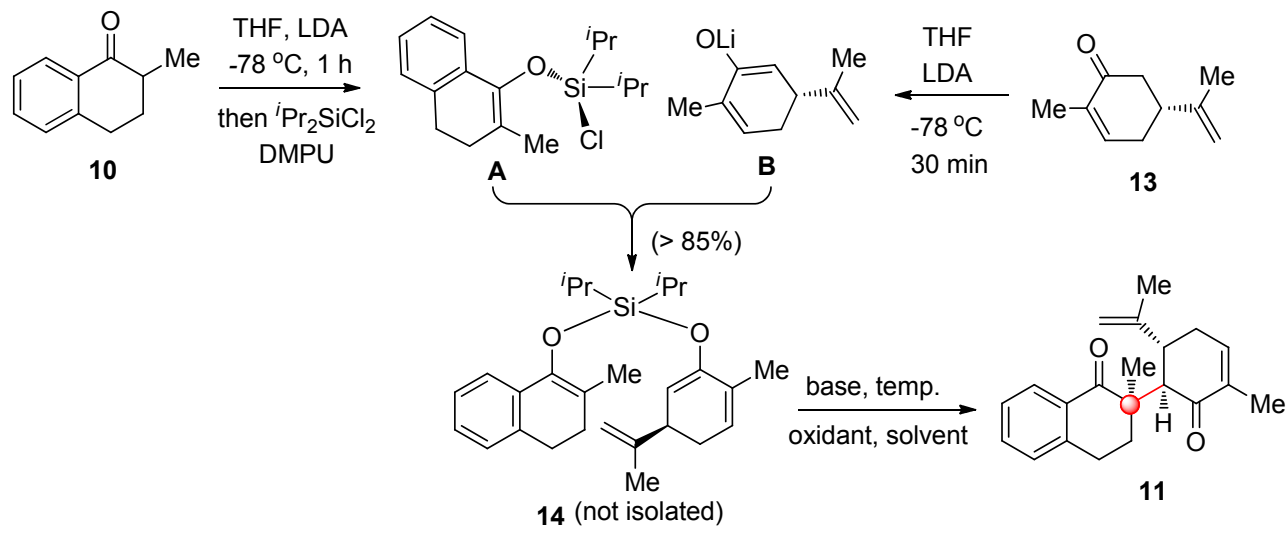

图式 3 反应前体 14 的合成

Scheme 3 Synthesis of reaction precursor 14

表 1 反应条件的优化 ${ }^{a}$

Table 1 Optimization of reaction conditions

\begin{tabular}{|c|c|c|c|c|c|}
\hline Entry & Base & Temp. $/{ }^{\circ} \mathrm{C}$ & Oxidant & Solvent & Yield $^{b} / \%$ \\
\hline 1 & None & -10 & CAN & $\mathrm{MeCN}$ & 45 \\
\hline 2 & $\mathrm{NaHCO}_{3}$ & -10 & CAN & $\mathrm{MeCN}$ & 50 \\
\hline 3 & $\mathrm{Cs}_{2} \mathrm{CO}_{3}$ & -10 & CAN & $\mathrm{MeCN}$ & 35 \\
\hline 4 & $\mathrm{~K}_{2} \mathrm{CO}_{3}$ & -10 & CAN & $\mathrm{MeCN}$ & 53 \\
\hline 5 & DTBP & -10 & CAN & $\mathrm{MeCN}$ & 55 \\
\hline 6 & DTBP & -10 & $\mathrm{FeCl}_{3}$ & $\mathrm{MeCN}$ & 0 \\
\hline 7 & DTBP & -10 & $\mathrm{Mn}(\mathrm{OAc})_{3} \cdot 2 \mathrm{H}_{2} \mathrm{O}$ & $\mathrm{MeCN}$ & 0 \\
\hline 8 & DTBP & -10 & $\mathrm{Cu}(\mathrm{OAc})_{2}$ & $\mathrm{MeCN}$ & $<5$ \\
\hline 9 & DTBP & -10 & $\mathrm{Cu}(\mathrm{OTf})_{2}$ & $\mathrm{MeCN}$ & 47 \\
\hline 10 & DTBP & -20 & CAN & $\mathrm{MeCN}$ & 55 \\
\hline 11 & DTBP & -30 & CAN & $\mathrm{MeCN}$ & 58 \\
\hline 12 & DTBP & -30 & CAN & $\operatorname{MeCN} / \mathrm{THF}(V: V=4: 1)$ & 60 \\
\hline
\end{tabular}

${ }^{a}$ Reaction conditions: 10 (0.12 mmol), 13 (1.0 equiv.), oxidant (2.0 equiv.) and base (3.0 equiv.) in anhydrous MeCN $(4.0 \mathrm{~mL})$ or MeCN/THF (5.0 mL). ${ }^{b}$ Isolated yield of 11 after flash column chromatography over two steps. $d r$ value was determined by ${ }^{1} \mathrm{H}$ NMR analysis of crude product. The stereochemistry was determinded by NOESY analysis. 
在得到了该反应的最优条件后, 我们开始进行底物 的拓展研究(表 2), 以验证该反应在非对映选择性构建 连续季碳中心和叔碳中心方面的有效性和普适性. 我们 首先改变底物 14 中的右侧片段, 通过选择不同环系的 酮包括几种简单的天然产物如 $(R)$-carvone, $(S)$-carvone, $(R)$-camphor 和 $(R)$-pulegone 与左侧片段 $\mathbf{1 0}$ 反应得到相 应的双烯醇硅醚底物, 之后在最优的反应条件下进行分 子内交叉氧化偶联反应, 实验结果表明其均能以较好的 收率和良好的非对映选择性提供相应的偶联产物 11, 12 和 15 20, 它们的相对立体化学通过 NOESY 进行确认; 其中, 偶联产物 15 的立体化学还通过 $\mathrm{X}$ 射线单晶衍射 进一步确认 ${ }^{[19]}$. 此外, 值得一提的是, 该反应还能以克

表 2 底物拓展 $\mathrm{I}^{a}$

Table 2 Substrate scope I

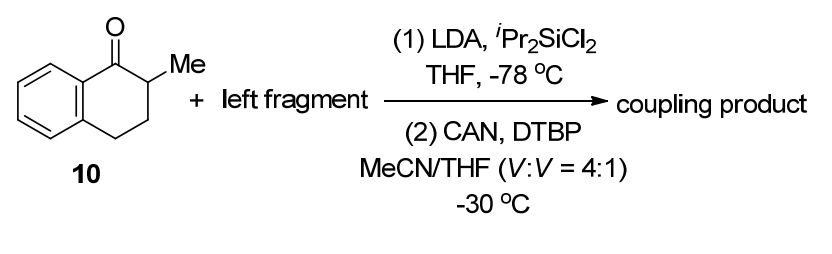

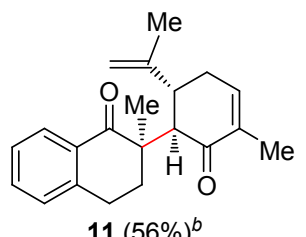

$11(56 \%)^{b}$

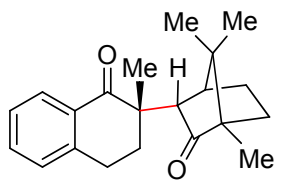

$15(43 \%)$

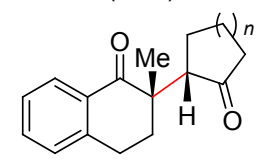

$17 n=1(58 \%, 15: 1 d r)$

$18 n=2(56 \%, 10: 1 d r)$

$19 n=3(59 \%, 16: 1 d r)$

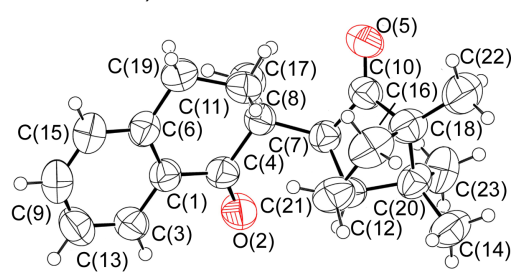

ORTEP of 15

(CDCC 1501822)

\footnotetext{
${ }^{a}$ The reaction was conducted on $0.66 \mathrm{mmol}$ scale. All yields were those of the isolated products over two steps. $d r$ value was determined by ${ }^{1} \mathrm{H}$ NMR or GC-MS analysis and the major diastereomer was shown. The stereochemistry was determined by either NOESY or X-ray analysis. ${ }^{b}$ Carried out on $1.26 \mathrm{~g}$ scale.
}

级别的规模进行, 并且产率无明显的下降 $(56 \%, 11)$.

为了进一步拓展底物的适用性，我们选取 $(R)$-香芹 酮(13)作为固定的右侧片段, 并与各种芳香酩(包括四氢 萗酮、狮满酮、色满酮)反应得到相应的双烯醇硅醚底 物, 之后在最优反应条件下进行分子内的交叉氧化偶联 反应, 如表 3 所示. 实验结果表明, 所有底物均能以较 好的收率和优异的非对映选择性 $(>20: 1 d r)$ 得到相应 偶联产物 21 31, 其相对立体化学通过 NOESY 进行确 认; 其中, 偶联产物 30 的立体化学还通过 X射线单晶衍 射进一步确认 ${ }^{[19]}$. 实验结果显示, 当苯环上具有供电子 基团(EDG)时 (22, 23，25 27)的产率要比具有吸电子基 团 $(E W G)$ 的底物 $(\mathbf{2 4}, \mathbf{2 8})$ 高, 我们推测可能是因为 $\mathrm{EDG}$ 更有利于反应过程所产生自由基中间体的加成过程 ${ }^{[20]}$, 但取代基的位置(邻、间、对位)对反应效果影响不大 $(25 \sim 27)$.

表 3 底物拓展 $\mathrm{II}^{a}$

Table 3 Substrate scope II

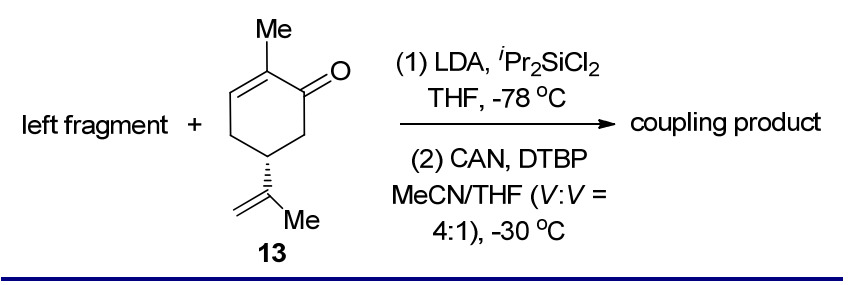<smiles>[R][R]1ccc2c(c1)CC1C(=O)C(C)=CC[C@H]1C2C(=C)C</smiles>

$22 \mathrm{R}=6-\mathrm{Me}(62 \%)$

$23 \mathrm{R}=6-\mathrm{MeO}(65 \%)$

$24 \mathrm{R}=6-\mathrm{F}(54 \%)^{b}$<smiles>C=C(C)[C@@H]1CC=C(C)C(=O)[C@H]1C1CCc2occc2C1=O</smiles>

$30(62 \%)$<smiles>C=C(C)[C@@H]1CC=C(C)C(=O)[C@@H]1C1COc2ccccc2C1=O</smiles>

31 (57\%)
$21 \mathrm{R}=\mathrm{H}(58 \%$

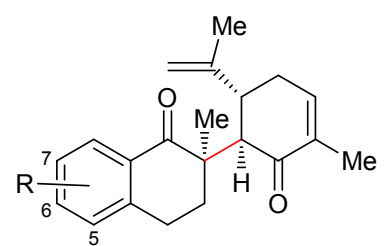

$25 \mathrm{R}=5-\mathrm{MeO}(64 \%)$

$26 \mathrm{R}=6-\mathrm{MeO}(59 \%)$

$27 \mathrm{R}=7-\mathrm{MeO}(65 \%)$

$28 \mathrm{R}=7-\mathrm{Br}(55 \%)$

$29 \mathrm{R}=5-\mathrm{Ph}(\mathrm{CO}) \mathrm{O}(60 \%)$

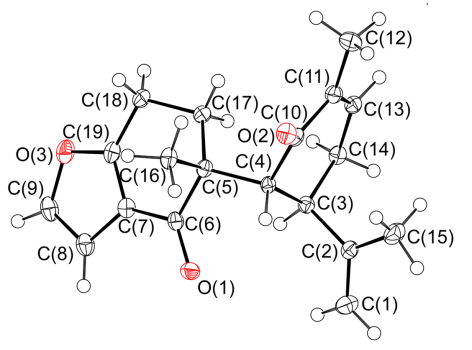

ORTEP of $\mathbf{3 0}$

(CDCC 1582641)

\footnotetext{
The reaction was conducted on $100 \mathrm{mg}$ scale. All yields were those of the isolated products over two steps. $d r$ value was determined by ${ }^{1} \mathrm{H}$ NMR analysis of crude product. The stereochemistry was determined by either NOESY or X-ray analysis. ${ }^{b}$ Carried out on $92 \mathrm{mg}$ scale.
} 
为了证明所发展的双烯醇硅醚化合物分子内交叉 氧化偶联反应对于控制反应非对映选择性的必要性, 我 们接着进行了分子间交叉氧化偶联反应作为对照实验. 根据 Frazier 和 Harlow ${ }^{[21]}$ 报道的条件, 四氢䒺酮底物 $\mathbf{1 0}$ 和 $(R)$-香芹酮 13 在 LDA 作碱的条件下首先形成烯醇负 离子, 之后利用 $\mathrm{FeCl}_{3}$ 作为单电子氧化剂进行分子间的 交叉氧化偶联反应(Scheme 4), 最终以 $9 \%$ 的产率得到交 叉偶联产物 11, 同时以 19\%的产率得到另一非对映异构 体 32, 其绝对立体化学通过 $\mathrm{X}$ 射线单晶衍射进行确认. 这一实验结果充分说明利用双烯醇硅醚底物能很好地 实现非对映选择性的交叉氧化偶联反应.<smiles>[M]C1CCc2ccccc2C1=O</smiles>

10

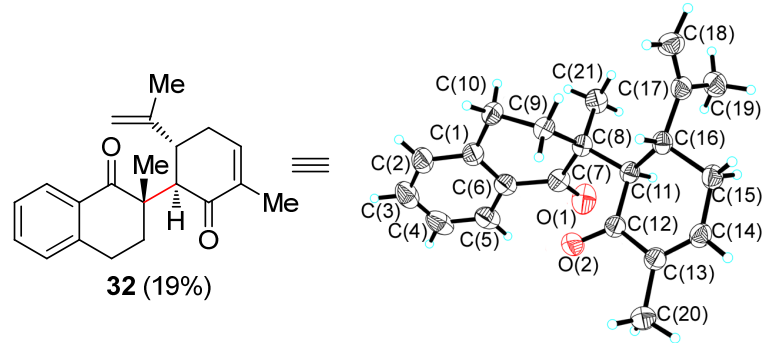

(CDCC 1861685)

图式 4 对照实验

Scheme 4 Control experiment

为进一步探究该反应的可能机理, 我们利用自由基 捕获剂 2,2,6,6-四甲基哌啶氧化物(TEMPO)进行了自由 基捕获实验(Scheme 5). 在标准的分子内交叉氧化偶联 反应条件下添加自由基捕获剂 TEMPO, 反应 $15 \mathrm{~min}$ 后
便以 $65 \%$ 的产率分离得到产物 33 , 而另一可能的产物 34 只能被高分辨质谱(HRMS)检测到。这一实验结果表 明，在该反应过程中，CAN 可能优先氧化双烯醇硅醚底 物 14 中的右侧片段.

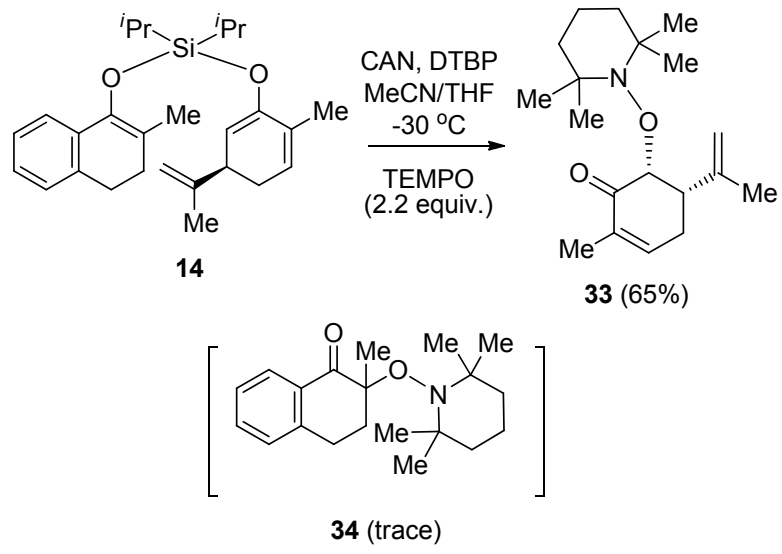

图式 5 TEMPO 自由基捕获实验

Scheme 5 Radical trapping experiment by TEMPO

基于以上实验结果以及前人报道的工作 ${ }^{[8,9]}$, 我们 提出了该反应可能的机理(Scheme 6). 首先, 在 CAN 存 在的条件下, 双烯醇硅醚底物 14 通过单电子转移过程 (SET)生成自由基中间体 $\mathbf{I I}^{[22]}$. 该自由基为缺电子物种, 故属于亲电性的自由基. 因此，通过其对左侧双键进行 自由基加成反应形成 $\mathrm{C}-\mathrm{C}$ 键并构建分子中的全碳季碳 立体中心，随后所得的自由基中间体 III 被另一分子的 CAN 氧化形成正离子中间体 IV 并得到最终产物 11. 由 于异丙烯基的立体位阻效应以及硅原子上取代基的偕 二甲基效应(Thorpe-Ingold effect ${ }^{[9 b]}$ ，反应的中间过渡 态倾向于采取 I 的方式，故能使该反应取得良好的非对 映选择性.

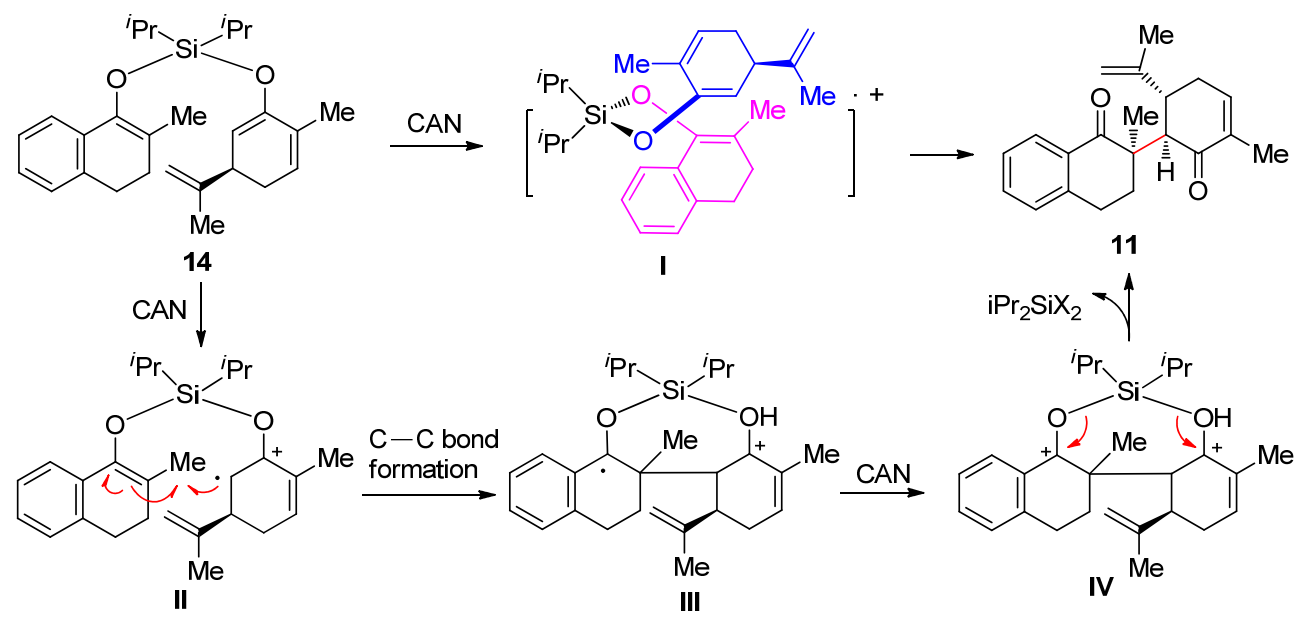

图式 6 可能的机理

Scheme 6 Proposed mechanism 


\section{2 结论}

基于前人研究工作的基础, 发展了一种利用 CAN 作为单电子氧化剂实现的双烯醇硅醚化合物分子内交 叉氧化偶联反应的方法学, 以较好的产率以及良好的非 对映选择性成功地实现了相邻连续季碳立体中心以及 叔碳立体中心的构建, 并对反应机理进行了初步的实验 研究. 该反应底物适用范围比较广, 且能实现规模化制 备, 这些特点为该方法学在复杂天然产物全合成中的应 用提供了可能.

\section{3 实验部分}

\section{1 仪器与试剂}

熔点值用 SGWX-4B 熔点仪测定(温度未校正); 旋 光值用 Horiba SEPA-300 旋光仪测得; 所有核磁共振谱 用 Brüker 公司 Advance 300 型核磁共振仪 $\left({ }^{1} \mathrm{H}\right.$ NMR 300 $\mathrm{MHz},{ }^{13} \mathrm{C}$ NMR $\left.75 \mathrm{MHz}\right) 、$ Advance 400 型核磁共振仪 $\left({ }^{1} \mathrm{H}\right.$ NMR $400 \mathrm{MHz},{ }^{13} \mathrm{C}$ NMR $100 \mathrm{MHz}$ ) 和 Advance 500 型核 磁共振仪 $\left({ }^{1} \mathrm{H}\right.$ NMR $500 \mathrm{MHz},{ }^{13} \mathrm{C}$ NMR $125 \mathrm{MHz}$ )测得, 使用氞代氯仿作为溶剂; 红外数据用 Shimadzu IR Prestige-21 傅里叶红外光谱仪 $(\mathrm{KBr}$ 压片)测得; 高分辨质谱 用 Brüker Apex IV RTMS 仪器测得; 反应检测用 0.25 mm 60F-254 的薄层色谱(TLC)硅胶板(青岛海洋化工厂). 柱层析用 200 300 目硅胶、中性或碱性氧化铝(青岛海 洋化工厂); 洗脱剂采用乙酸乙酯和石油梄(b.p. 60 $90{ }^{\circ} \mathrm{C}$ ); 所用其他溶剂均用标准方法纯化后使用.

\section{2 实验方法}

\subsection{1 反应前体双烯醇硅醚底物的合成}

将左侧片段四氢萗酮底物(1.0 equiv)溶解在四氢呋 喃中, 在 $-78{ }^{\circ} \mathrm{C}$ 和氩气氛围下逐滴加入二异丙基氨基 锂 ( $2 \mathrm{~mol} \cdot \mathrm{L}^{-1}$, 溶于 THF, 1.1 equiv.), 在此温度下搅拌反 应 $1 \mathrm{~h}$ 得到 solution I; 而在另一反应容器中将二异丙基 二氯化硅(1.1 equiv.)和 $N, N$-二甲基丙烯基脲(DMPU, 3.0 equiv.) 溶解在四氢呋喃中, 然后在 $-78{ }^{\circ} \mathrm{C}$ 和氩气氛围 下将 solution I 通过导管逐滴加入该反应体系中并在此 温度下继续摚拌反应 $1 \mathrm{~h}$ 得到 solution II; 此外, 将右侧 片段 (1.0 equiv)溶解在四氢呋喃中, 在 $-78{ }^{\circ} \mathrm{C}$ 和氩气氛 围下逐滴加入二异丙基氨基锂 $\left(2 \mathrm{~mol} \cdot \mathrm{L}^{-1}\right.$, 溶于 $\mathrm{THF}$, 1.1 equiv.), 在此温度下摚拌反应 $30 \mathrm{~min}$ 得到 solution III; 随后在 $-78{ }^{\circ} \mathrm{C}$ 和氩气氛围下将 solution III 通过导 管逐滴加入到 solution II 中, 并在此温度下继续摚拌反 应 $30 \mathrm{~min}$ 至 $1 \mathrm{~h}$, 薄层色谱法监测反应完毕后, 用 $\mathrm{pH}$ 为 7.0 的缓冲液 (由磷酸二氢钾和磷酸氢二钾组成) 淬灭反 应, 然后用乙酸乙酯萃取水相, 合并所得有机相并用无 水硫酸钠干燥. 减压浓缩后, 残余物经 $200 \sim 300$ 目中性
或者碱性氧化铝填充的短色谱柱分离(填充高度 $5 \mathrm{~cm}$, 正已烷作洗脱剂), 以大于 $85 \%$ 的收率( ${ }^{1} \mathrm{H}$ NMR 确定)得 到相应的双烯醇硅醚粗产物(由于部分双烯醇硅醚底物 不稳定, 故无需进一步纯化而直接旋干溶剂用于下一步 反应).

$(R)$-二异丙基- $O, O$-(2-甲基-3,4-二氢萘)(6-甲基-3-异 丙烯基-1,5-双烯)硅烷(14)：无色油状液体 $\left[R_{\mathrm{f}}=0.94\right.$, $V($ 正己烷 $) ： V$ (乙酸乙酯 $)=16 ： 1] .{ }^{1} \mathrm{H}$ NMR $(500 \mathrm{MHz}$, $\left.\mathrm{CDCl}_{3}\right) \delta: 7.51(\mathrm{~d}, J=7.7 \mathrm{~Hz}, 1 \mathrm{H}), 7.17(\mathrm{td}, J=7.9,2.2$ $\mathrm{Hz}, 1 \mathrm{H}), 7.13 \sim 7.06(\mathrm{~m}, 2 \mathrm{H}), 5.60(\mathrm{~s}, 1 \mathrm{H}), 5.06$ (d, $J=3.6$ $\mathrm{Hz}, 1 \mathrm{H}), 4.78(\mathrm{~d}, J=1.0 \mathrm{~Hz}, 1 \mathrm{H}), 4.71(\mathrm{~s}, 1 \mathrm{H}), 3.00 \sim 3.06$ (m, 1H), 2.74 (t, $J=7.9 \mathrm{~Hz}, 2 \mathrm{H}), 2.27$ (t, $J=7.9 \mathrm{~Hz}, 2 \mathrm{H})$, $2.21 \sim 2.10(\mathrm{~m}, 2 \mathrm{H}), 1.91(\mathrm{~s}, 3 \mathrm{H}), 1.77(\mathrm{~s}, 3 \mathrm{H}), 1.72(\mathrm{~s}$, $3 \mathrm{H}), 1.29 \sim 1.18(\mathrm{~m}, 2 \mathrm{H}), 1.12(\mathrm{~d}, J=7.2 \mathrm{~Hz}, 12 \mathrm{H}) ;{ }^{13} \mathrm{C}$ NMR $\left(125 \mathrm{MHz}, \mathrm{CDCl}_{3}\right) \delta: 149.2,148.4,142.5,135.8$, $134.1,131.8,126.6,126.2,126.1,123.2,121.4,116.4$, 110.0, 105.6, 77.3, 77.0, 76.7, 42.0, 29.4, 28.6, 28.1, 20.6, 17.6, 17.5, 17.5, 17.4, 17.4, 17.3, 13.2, 13.0; IR (film) $v_{\max }$ : 2981, 1730, 1658, 1554, 1258, 1131, 969, 886, $732 \mathrm{~cm}^{-1}$; HRMS (ESI) calcd for $\mathrm{C}_{27} \mathrm{H}_{39} \mathrm{O}_{2} \mathrm{Si}[\mathrm{M}+\mathrm{H}]^{+} 423.2714$, found 423.2713 .

\subsubsection{CAN 促进的分子内交叉氧化偶联反应}

将硝酸铈铵(CAN, 2.0 equiv.) 和 2,6-二叔丁基吡啶 (DTBP, 2.0 equiv.) 溶解在乙腈 $(4.0 \mathrm{~mL})$ 中, 然后在一 $30{ }^{\circ} \mathrm{C}$ 和氩气氛围下通过导管加入双烯醇硅醚底物 $(1.0$ equiv., 溶解在 $1.0 \mathrm{~mL}$ 的四氢呋喃中), 在此温度下搅拌 反应 $15 \mathrm{~min}$ 后, 用饱和氯化铵水溶液 $(3.0 \mathrm{~mL}$ )淬灭反应, 用乙酸乙酯 $(5.0 \mathrm{~mL} \times 3)$ 萃取水相, 合并所得有机相用无 水硫酸钠干燥. 减压浓缩后, 残余物经快速柱色谱分离 $[V($ 正己烷 $): V$ (乙酸乙酯 $)=25: 1 \rightarrow 20 ： 1]$ 得到相应的 偶联产物. $d r$ 值由粗产物的 ${ }^{1} \mathrm{H}$ NMR 或者 GC-MS 确定 可得.

( $R$ )-2- 甲基-2-(( $1 R, 6 R)$-3- 甲基-2-氧-6-异丙烯基)-

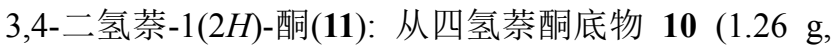
$7.92 \mathrm{mmol}$ )出发, 以 $56 \%$ 产率得到 $1.37 \mathrm{~g}$ 浅黄色固体 $\left[R_{\mathrm{f}}=0.37, V(\right.$ 正己烷 $): V($ 乙酸乙酯 $\left.)=8: 1\right]$. m.p. $103 \sim$ $106{ }^{\circ} \mathrm{C} ;[\alpha]_{\mathrm{D}}^{20}+165\left(c 0.5, \mathrm{CHCl}_{3}\right) ;{ }^{1} \mathrm{H}$ NMR $(400 \mathrm{MHz}$, $\left.\mathrm{CDCl}_{3}\right) \delta: 8.05(\mathrm{dd}, J=7.9,1.2 \mathrm{~Hz}, 1 \mathrm{H}), 7.45(\mathrm{td}, J=7.5$, $1.4 \mathrm{~Hz}, 1 \mathrm{H}), 7.32(\mathrm{t}, J=7.3 \mathrm{~Hz}, 1 \mathrm{H}), 7.19(\mathrm{~d}, J=7.6 \mathrm{~Hz}$, $1 \mathrm{H}), 6.61(\mathrm{~s}, 1 \mathrm{H}), 4.74 \sim 4.54(\mathrm{~m}, 2 \mathrm{H}), 3.06(\mathrm{~d}, J=2.4 \mathrm{~Hz}$, $1 \mathrm{H}), 2.99 \sim 2.89(\mathrm{~m}, 2 \mathrm{H}), 2.90 \sim 2.82(\mathrm{~m}, 1 \mathrm{H}), 2.72 \sim 2.60$ $(\mathrm{m}, 1 \mathrm{H}), 2.43(\mathrm{~m}, 1 \mathrm{H}), 2.38 \sim 2.25(\mathrm{~m}, 1 \mathrm{H}), 1.94 \sim 2.00$ (m, $1 \mathrm{H}), 1.78(\mathrm{dd}, J=3.7,1.7 \mathrm{~Hz}, 3 \mathrm{H}), 1.71(\mathrm{~s}, 3 \mathrm{H}), 1.26$ (s, 3H); ${ }^{13} \mathrm{C}$ NMR $\left(100 \mathrm{MHz}, \mathrm{CDCl}_{3}\right) \delta: 200.5,199.9$, $149.0,142.5,142.0,136.7,132.9,132.3,128.4,128.3$, 
126.9, 111.4, 77.3, 77.0, 76.7, 53.3, 49.2, 41.7, 31.9, 29.8, 25.2, 21.0, 20.92, 16.3; IR (film) $v_{\max }: 2922,1738,1660$, 1449, 1225, 1192, 989, 889, $745 \mathrm{~cm}^{-1}$; HRMS (ESI) calcd for $\mathrm{C}_{21} \mathrm{H}_{25} \mathrm{O}_{2}[\mathrm{M}+\mathrm{H}]^{+}$309.1849, found 309.1850.

(S)-2-甲基-2-((1S,6S)-3-甲基-2-氧-6-异丙烯基)-3,4二氢萗-1 $(2 H)$-酮 $(12)$ : 从四氢菜酮底物 $10(0.66 \mathrm{mmol})$ 出发, 以 $63 \%$ 产率得到 $128 \mathrm{mg}$ 浅黄色固体 $\left[R_{\mathrm{f}}=0.37\right.$, $V($ 正己烷 $): V($ 乙酸乙酯 $)=8 ： 1)$. m.p. $103 \sim 106{ }^{\circ} \mathrm{C}$; $[\alpha]_{\mathrm{D}}^{20}-155\left(c 0.5, \mathrm{CHCl}_{3}\right) ;{ }^{1} \mathrm{H}$ NMR $\left(400 \mathrm{MHz}, \mathrm{CDCl}_{3}\right)$ $\delta: 8.05(\mathrm{dd}, J=7.9,1.2 \mathrm{~Hz}, 1 \mathrm{H}), 7.45(\mathrm{td}, J=7.5,1.4 \mathrm{~Hz}$, $1 \mathrm{H}), 7.32$ (t, $J=7.3 \mathrm{~Hz}, 1 \mathrm{H}), 7.19$ (d, $J=7.6 \mathrm{~Hz}, 1 \mathrm{H}), 6.61$ (s, 1H), 4.68 (dd, $J=2.7,1.2 \mathrm{~Hz}, 2 \mathrm{H}), 3.06$ (d, $J=2.4 \mathrm{~Hz}$, $2 \mathrm{H}), 2.98 \sim 2.80(\mathrm{~m}, 3 \mathrm{H}), 2.76 \sim 2.56(\mathrm{~m}, 1 \mathrm{H}), 2.43(\mathrm{~m}$, $1 \mathrm{H}), 2.39 \sim 2.28(\mathrm{~m}, 1 \mathrm{H}), 2.03 \sim 1.94(\mathrm{~m}, 1 \mathrm{H}), 1.78(\mathrm{~d}, J=$ $1.9 \mathrm{~Hz}, 3 \mathrm{H}), 1.72(\mathrm{~s}, 3 \mathrm{H}), 1.26(\mathrm{~s}, 3 \mathrm{H}) ;{ }^{13} \mathrm{C}$ NMR $(100$ $\left.\mathrm{MHz}, \mathrm{CDCl}_{3}\right) \delta: 200.5,199.9,149.0,142.5,142.0,136.7$, $132.9,132.3,128.4,128.3,126.9,111.4,77.3,77.0,76.7$, $53.3,49.2,41.8,31.9,29.8,25.2,21.0,20.9,16.2$; IR (film) $v_{\text {max }}: 2925,1736,1665,1453,1228,1195,990,895$, $750 \mathrm{~cm}^{-1}$; HRMS (APCI) calcd for $\mathrm{C}_{21} \mathrm{H}_{25} \mathrm{O}_{2}[\mathrm{M}+\mathrm{H}]^{+}$: 309.1849, found 309.1849 .

(S)-2-甲基-2-( $S$ )-2-甲基-2-(( $1 S, 2 S, 4 R)-4,7,7$-三甲基3 -并环[2.2.1]庚烷)-3,4-二氢萘-1 $(2 H)$-酮 $(15)$ : 从四氢萘 酮底物 10 (0.66 mmol) 出发, 以 $43 \%$ 产率得到 $88.3 \mathrm{mg}$ 白 色固体 $15\left[R_{\mathrm{f}}=0.37, V(\right.$ 正己烷 $): V($ 乙酸乙酯 $\left.)=8: 1\right)$. m.p. $108 \sim 116{ }^{\circ} \mathrm{C} .[\alpha]_{\mathrm{D}}^{20}+95\left(c \quad 0.1, \mathrm{CHCl}_{3}\right) ;{ }^{1} \mathrm{H}$ NMR $\left(400 \mathrm{MHz}, \mathrm{CDCl}_{3}\right) \delta: 8.02(\mathrm{~d}, J=7.8 \mathrm{~Hz}, 1 \mathrm{H}), 7.46$ (td, $J=7.5,1.1 \mathrm{~Hz}, 1 \mathrm{H}), 7.31(\mathrm{t}, J=7.6 \mathrm{~Hz}, 1 \mathrm{H}), 7.22$ (d, $J=$ $7.6 \mathrm{~Hz}, 1 \mathrm{H}), 3.11(\mathrm{~m}, 1 \mathrm{H}), 2.89 \sim 2.81(\mathrm{~m}, 2 \mathrm{H}), 2.42(\mathrm{t}$, $J=3.9 \mathrm{~Hz}, 1 \mathrm{H}), 2.23(\mathrm{td}, J=12.9,4.9 \mathrm{~Hz}, 1 \mathrm{H}), 2.13(\mathrm{~m}$, $1 \mathrm{H}), 1.71 \sim 1.61(\mathrm{~m}, 2 \mathrm{H}), 1.52(\mathrm{dd}, J=19.9,9.6 \mathrm{~Hz}, 2 \mathrm{H})$, $1.45(\mathrm{~s}, 3 \mathrm{H}), 0.97(\mathrm{~s}, 3 \mathrm{H}), 0.94(\mathrm{~s}, 3 \mathrm{H}), 0.90(\mathrm{~s}, 3 \mathrm{H}) ;{ }^{13} \mathrm{C}$ NMR (100 MHz, $\left.\mathrm{CDCl}_{3}\right) \delta: 219.1,200.9,142.7,133.1$, 132.2, 128.4, 128.1, 126.7, 77.3, 77.0, 76.7, 59.5, 57.2, 47.7, 45.9, 45.6, 32.8, 30.5, 25.8, 23.1, 22.3, 20.0, 19.0, 9.8; IR (film) $v_{\max }: 2923,1733,1680,1568,1235,1189$, $945,897,740 \mathrm{~cm}^{-1}$; HRMS (ESI) calcd for $\mathrm{C}_{21} \mathrm{H}_{27} \mathrm{O}_{2}[\mathrm{M}+$ $\mathrm{H}]^{+}$311.2006, found 311.2009.

(R)-2-甲基-2-((1R,6R)-3-甲基-2-氧-6-甲基)-3,4-二 氢萗-1 $(2 H)$-酮 $(\mathbf{1 6})$ : 从四氢萗酮底物 $10(0.66 \mathrm{mmol})$ 出 发, 以 $58 \%$ 产率得到 $119 \mathrm{mg}$ 无色油状液体 $16\left[R_{\mathrm{f}}=0.37\right.$, $V($ 正己烷 $): V($ 乙酸乙酯 $)=8: 1), d r=13: 1$, 这里仅展 示主要优势构型产物 16. $[\alpha]_{\mathrm{D}}^{20}+265\left(c 0.6, \mathrm{CHCl}_{3}\right) ;{ }^{1} \mathrm{H}$ NMR (400 MHz, $\left.\mathrm{CDCl}_{3}\right) \delta: 8.01(\mathrm{~d}, J=7.8 \mathrm{~Hz}, 1 \mathrm{H}), 7.44$ (td, $J=7.4,1.2 \mathrm{~Hz}, 1 \mathrm{H}), 7.30(\mathrm{t}, J=7.4 \mathrm{~Hz}, 1 \mathrm{H}), 7.20$ (d,
$J=7.5 \mathrm{~Hz}, 1 \mathrm{H}), 3.03 \sim 2.90(\mathrm{~m}, 2 \mathrm{H}), 2.74(\mathrm{t}, J=8.4 \mathrm{~Hz}$, $1 \mathrm{H}), 2.54(\mathrm{~d}, J=15.4 \mathrm{~Hz}, 1 \mathrm{H}), 2.43$ (d, $J=9.9 \mathrm{~Hz}, 1 \mathrm{H})$, $2.36 \sim 2.25(\mathrm{~m}, 1 \mathrm{H}), 2.25 \sim 2.13(\mathrm{~m}, 1 \mathrm{H}), 2.06 \sim 1.92(\mathrm{~m}$, $1 \mathrm{H}), 1.87(\mathrm{~s}, 3 \mathrm{H}), 1.84 \sim 1.76(\mathrm{~m}, 1 \mathrm{H}), 1.72(\mathrm{~s}, 3 \mathrm{H}), 1.42 \sim$ $1.31(\mathrm{~m}, 1 \mathrm{H}), 1.27(\mathrm{~s}, 3 \mathrm{H}), 1.08(\mathrm{~d}, J=6.7 \mathrm{~Hz}, 3 \mathrm{H}) ;{ }^{13} \mathrm{C}$ NMR $\left(100 \mathrm{MHz}, \mathrm{CDCl}_{3}\right) \delta: 207.3,201.1,142.5,139.4$, $134.1,133.0,132.5,128.4,128.3,126.9,77.4,77.0,76.8$, $62.0,48.3,33.3,31.9,31.5,27.8,25.5,23.8,22.1,21.5$, 21.1; IR (film) $v_{\max }: 2924,1754,1674,1455,1220,1186$, $987,879,740 \mathrm{~cm}^{-1}$; HRMS (ESI) calcd for $\mathrm{C}_{21} \mathrm{H}_{27} \mathrm{O}_{2}[\mathrm{M}+$ $\mathrm{H}]^{+}$311.2006, found 311.2008.

2-甲基-2-氧环戊基-3,4-二氢萗-1(2H)-酮(17)：从四 氢菜酮底物 10 (0.66 mmol)出发, 以 $58 \%$ 产率得到 92.6 $\mathrm{mg}$ 无色油状液体 $\left[R_{\mathrm{f}}=0.38, V\right.$ (正己烷 $): V($ 乙酸乙酯 $)=$ $8: 1] . d r=15: 1$, 这里仅展示主要优势构型产物 $17 .{ }^{1} \mathrm{H}$ NMR (400 MHz, $\left.\mathrm{CDCl}_{3}\right) \delta: 7.99(\mathrm{dd}, J=7.9,1.2 \mathrm{~Hz}, 1 \mathrm{H})$, $7.46(\mathrm{td}, J=7.5,1.4 \mathrm{~Hz}, 1 \mathrm{H}), 7.33 \sim 7.27(\mathrm{~m}, 1 \mathrm{H}), 7.22(\mathrm{~d}$, $J=7.7 \mathrm{~Hz}, 1 \mathrm{H}), 3.14$ (ddd, $J=12.3,9.8,5.1 \mathrm{~Hz}, 1 \mathrm{H}), 2.89$ (ddd, $J=17.4,5.1,3.0 \mathrm{~Hz}, 1 \mathrm{H}), 2.64(\mathrm{td}, J=12.8,5.2 \mathrm{~Hz}$, $1 \mathrm{H}), 2.45 \sim 2.35(\mathrm{~m}, 1 \mathrm{H}), 2.25(\mathrm{~m}, 3 \mathrm{H}), 2.04$ (ddd, $J=$ $13.3,7.1,4.5 \mathrm{~Hz}, 2 \mathrm{H}), 1.84(\mathrm{~m}, 1 \mathrm{H}), 1.78 \sim 1.68(\mathrm{~m}, 1 \mathrm{H})$, $1.38(\mathrm{~s}, 3 \mathrm{H}) ;{ }^{13} \mathrm{C}$ NMR (100 MHz, $\left.\mathrm{CDCl}_{3}\right) \delta: 220.3,201.6$, $142.8,133.2$, 131.7, 128.6, 128.0, 126.6, 77.3, 77.0, 76.7, 55.6, 46.9, 39.7, 32.0, 27.1, 25.3, 20.6, 20.2; IR (film) $v_{\max }$ : 2945, 1767, 1687, 1535, 1278, 1154, 957, 824, $717 \mathrm{~cm}^{-1}$; HRMS (ESI) calcd for $\mathrm{C}_{16} \mathrm{H}_{19} \mathrm{O}_{2}[\mathrm{M}+\mathrm{H}]^{+}$243.1380, found 243.1380 .

2-甲基-2-氧环己基-3,4-二氢䒺-1(2H)-酮(18)：从四 氢菜酮底物 10 (0.66 mmol) 出发, 以 $56 \%$ 产率得到 94.6 $\mathrm{mg}$ 无色油状液体 $\left[R_{\mathrm{f}}=0.42, V\right.$ (正己烷 $): V($ 乙酸乙酯 $)=$ $8: 1], d r=10: 1$, 这里仅展示主要优势构型产物 $18 .{ }^{1} \mathrm{H}$ NMR (400 MHz, $\left.\mathrm{CDCl}_{3}\right) \delta: 8.04(\mathrm{dd}, J=7.9,1.1 \mathrm{~Hz}, 1 \mathrm{H})$, $7.45(\mathrm{td}, J=7.5,1.4 \mathrm{~Hz}, 1 \mathrm{H}), 7.31(\mathrm{t}, J=7.6 \mathrm{~Hz}, 1 \mathrm{H}), 7.22$ $(\mathrm{d}, J=7.6 \mathrm{~Hz}, 1 \mathrm{H}), 3.19 \sim 3.05(\mathrm{~m}, 1 \mathrm{H}), 3.00(\mathrm{dd}, J=12.6$, $4.4 \mathrm{~Hz}, 1 \mathrm{H}), 2.83$ (dt, $J=17.1,3.8 \mathrm{~Hz}, 1 \mathrm{H}), 2.36$ (dd, $J=$ $10.4,4.7 \mathrm{~Hz}, 2 \mathrm{H}), 2.24 \sim 2.18(\mathrm{~m}, 2 \mathrm{H}), 2.15 \sim 2.05(\mathrm{~m}$, $2 \mathrm{H}), 1.94 \sim 1.66(\mathrm{~m}, 4 \mathrm{H}), 1.30(\mathrm{~s}, 3 \mathrm{H}) ;{ }^{13} \mathrm{C}$ NMR $(100$ $\left.\mathrm{MHz}, \mathrm{CDCl}_{3}\right) \delta: 211.8,202.0,143.0,133.1,132.1,128.6$, $128.1,126.7,77.4,77.0,76.8,58.0,46.3,43.9,31.1,30.6$, 28.4, 26.2, 25.4, 20.1; IR (film) $v_{\max }: 2931,17.3,1679$, 1564, 1223, 1168, 963, 842, $740 \mathrm{~cm}^{-1}$; HRMS (ESI) calcd for $\mathrm{C}_{17} \mathrm{H}_{20} \mathrm{O}_{2} \mathrm{Na}[\mathrm{M}+\mathrm{Na}]^{+}$279.1356, found 279.1356.

2-甲基-2-氧环庚基-3,4-二氢菜- $1(2 H)$-酮(19): 从四 氢菜酮底物 10 (0.66 mmol) 出发, 以 59\%产率得到 105 $\mathrm{mg}$ 无色油状液体 $\left[R_{\mathrm{f}}=0.37, V\right.$ (正己烷) $: V$ (乙酸乙酯 $)=$ 
$8: 1], d r=16: 1$, 这里仅展示主要优势构型产物 $19 .{ }^{1} \mathrm{H}$ NMR (400 MHz, $\left.\mathrm{CDCl}_{3}\right) \delta: 8.03(\mathrm{dd}, J=7.9,1.1 \mathrm{~Hz}, 1 \mathrm{H})$, $7.46(\mathrm{td}, J=7.5,1.4 \mathrm{~Hz}, 1 \mathrm{H}), 7.31(\mathrm{t}, J=7.6 \mathrm{~Hz}, 1 \mathrm{H}), 7.21$ $(\mathrm{d}, J=7.6 \mathrm{~Hz}, 1 \mathrm{H}), 3.35$ (dd, $J=10.4,2.6 \mathrm{~Hz}, 1 \mathrm{H}), 3.00 \sim$ $3.08(\mathrm{~m}, 1 \mathrm{H}), 2.87(\mathrm{dt}, J=17.3,4.4 \mathrm{~Hz}, 1 \mathrm{H}), 2.62 \sim 2.52$ (m, $1 \mathrm{H}), 2.51 \sim 2.43(\mathrm{~m}, 1 \mathrm{H}), 2.43 \sim 2.34(\mathrm{~m}, 1 \mathrm{H}), 2.18$ (ddd, $J=13.6,11.8,4.9 \mathrm{~Hz}, 1 \mathrm{H}), 1.90$ (dd, $J=8.5,6.8$ $\mathrm{Hz}, 3 \mathrm{H}), 1.80 \sim 1.57(\mathrm{~m}, 2 \mathrm{H}), 1.52 \sim 1.36(\mathrm{~m}, 2 \mathrm{H}), 1.24(\mathrm{~s}$, $3 \mathrm{H}) ;{ }^{13} \mathrm{C}$ NMR $\left(100 \mathrm{MHz}, \mathrm{CDCl}_{3}\right) \delta: 215.3,201.5,142.9$, 133.1, 131.8, 128.5, 128.1, 126.7, 77.3, 77.0, 76.8, 55.5, 48.5, 45.0, 29.9, 29.4, 29.0, 28.1, 25.0, 24.3, 19.7; IR (film) $v_{\max }: 2922,1775,1660,1554,1265,1148,954,887$, $712 \mathrm{~cm}^{-1}$; HRMS (ESI) calcd for $\mathrm{C}_{18} \mathrm{H}_{23} \mathrm{O}_{2}[\mathrm{M}+\mathrm{H}]^{+}$ 271.1693, found 271.1695.

2- 甲基-3,3',4,4'-四氢-[2,2'-联菜]-1,1'( $\left.2 H, 2^{\prime} H\right)$-二酮 (20): 从四氢萗酮底物 $10(0.66 \mathrm{mmol})$ 出发, 以 $52 \%$ 产率 得到 $104 \mathrm{mg}$ 无色油状液体 $\left[R_{\mathrm{f}}=0.42, V\right.$ (正己烷) : $V($ 乙 酸乙酯) $=8: 1] .{ }^{1} \mathrm{H}$ NMR $\left(400 \mathrm{MHz}, \mathrm{CDCl}_{3}\right) \delta: 8.07(\mathrm{~d}$, $J=6.8 \mathrm{~Hz}, 1 \mathrm{H}), 7.99 \sim 7.94(\mathrm{~m}, 1 \mathrm{H}), 7.46(\mathrm{qd}, J=7.5,1.3$ $\mathrm{Hz}, 2 \mathrm{H}), 7.32(\mathrm{dd}, J=13.2,5.7 \mathrm{~Hz}, 2 \mathrm{H}), 7.29 \sim 7.21(\mathrm{~m}$, 2H), $3.27 \sim 2.85$ (m, 5H), 2.47 (td, $J=12.7,5.0 \mathrm{~Hz}, 1 \mathrm{H}$ ), 2.27 (dq, $J=5.3,4.1 \mathrm{~Hz}, 2 \mathrm{H}), 2.19 \sim 2.07$ (m, $1 \mathrm{H}), 1.46$ (s, $3 \mathrm{H}) ;{ }^{13} \mathrm{C}$ NMR (125 MHz, $\left.\mathrm{CDCl}_{3}\right) \delta: 202.1,199.1,143.5$, $142.7,133.9,133.0,133.0,132.2,128.5,128.5,128.1$, 127.3, 126.7, 126.5, 77.3, 77.00, 76.8, 55.1, 47.4, 31.4, 30.4, 27.0, 25.5, 20.4; IR (film) $v_{\text {max }}$ : 2990, 1754, 1247, $1053,749,764 \mathrm{~cm}^{-1}$; HRMS (ESI) calcd for $\mathrm{C}_{21} \mathrm{H}_{20} \mathrm{O}_{2} \mathrm{Na}$ $[\mathrm{M}+\mathrm{Na}]^{+}$327.1356, found 327.1355.

( $R$ )-2- 甲基 -2-(( $1 R, 6 R)$-3- 甲 基 -2-氧 -6- 异丙烯 基)-3,4-狮-1(2H)-酮(21): 从 2-甲基-1-茚满酮(100 mg)出 发, 以 $58 \%$ 产率得到 $116 \mathrm{mg}$ 无色油状液体 $\left[R_{\mathrm{f}}=0.38\right.$, $V($ 正己烷 $): V($ 乙酸乙酯 $)=8: 1] .[\alpha]_{\mathrm{D}}^{20}+150(c \quad 0.4$, $\left.\mathrm{CHCl}_{3}\right) ;{ }^{1} \mathrm{H}$ NMR $\left(400 \mathrm{MHz}, \mathrm{CDCl}_{3}\right) \delta: 7.71$ (d, $J=7.5$ $\mathrm{Hz}, 1 \mathrm{H}), 7.57(\mathrm{td}, J=7.5,1.2 \mathrm{~Hz}, 1 \mathrm{H}), 7.44 \sim 7.32(\mathrm{~m}$, 2H), $6.56(\mathrm{~d}, J=3.2 \mathrm{~Hz}, 1 \mathrm{H}), 4.58 \sim 4.55(\mathrm{~m}, 1 \mathrm{H}), 4.49$ (s, $1 \mathrm{H}), 3.42(\mathrm{~d}, J=17.6 \mathrm{~Hz}, 1 \mathrm{H}), 2.92(\mathrm{~d}, J=4.6 \mathrm{~Hz}, 1 \mathrm{H})$, 2.87 (d, $J=17.6 \mathrm{~Hz}, 1 \mathrm{H}), 2.76(\mathrm{dd}, J=10.2,4.8 \mathrm{~Hz}, 1 \mathrm{H})$, $2.37 \sim 2.29(\mathrm{~m}, 2 \mathrm{H}), 1.67(\mathrm{~d}, J=0.5 \mathrm{~Hz}, 3 \mathrm{H}), 1.65$ (d, $J=$ $1.5 \mathrm{~Hz}, 3 \mathrm{H}), 1.37$ (s, 3H); ${ }^{13} \mathrm{C}$ NMR (100 MHz, $\left.\mathrm{CDCl}_{3}\right) \delta$ : $208.8,199.8,152.2,147.3,142.5,136.3,135.9,134.7$, 127.5, 126.4, 124.1, 112.7, 77.3, 77.0, 76.7, 55.8, 51.5, 43.4, 38.8, 29.1, 25.2, 20.8, 16.0; IR (film) $v_{\max }: 2927$, 1708, 1660, 1578, 1279, 1192, 958, 878, $748 \mathrm{~cm}^{-1}$; HRMS (ESI) calcd for $\mathrm{C}_{20} \mathrm{H}_{23} \mathrm{O}_{2}[\mathrm{M}+\mathrm{H}]^{+}$295.1693, found 295.1693.
( $R$ )-2,6-二甲基-2-(( $1 R, 6 R)$-3- 甲基-2-氧-6-异丙烯 基)-3,4-二氢狮- $1(2 H)$-酮(22): 从 2,6-二甲基-1-苑满酮 $(100 \mathrm{mg})$ 出发, 以 $62 \%$ 产率得 $118 \mathrm{mg}$ 无色油状液体 $\left[R_{\mathrm{f}}=0.39, V\right.$ (正己烷) $: V$ (乙酸乙酯 $\left.)=8: 1\right] .[\alpha]_{\mathrm{D}}^{20}+130$ (c $\left.0.3, \mathrm{CHCl}_{3}\right) ;{ }^{1} \mathrm{H}$ NMR $\left(400 \mathrm{MHz}, \mathrm{CDCl}_{3}\right) \delta: 7.51(\mathrm{~s}$, 1H), 7.39 (dd, $J=7.8,1.2 \mathrm{~Hz}, 1 \mathrm{H}), 7.28(\mathrm{~d}, J=7.8 \mathrm{~Hz}$, $1 \mathrm{H}), 6.56(\mathrm{t}, J=3.0 \mathrm{~Hz}, 1 \mathrm{H}), 4.60 \sim 4.55(\mathrm{~m}, 1 \mathrm{H}), 4.50(\mathrm{~s}$, $1 \mathrm{H}), 3.36(\mathrm{~d}, J=17.5 \mathrm{~Hz}, 1 \mathrm{H}), 2.90(\mathrm{~d}, J=4.3 \mathrm{~Hz}, 1 \mathrm{H})$, 2.79 (d, $J=17.5 \mathrm{~Hz}, 1 \mathrm{H}), 2.71(\mathrm{dd}, J=10.0,4.3 \mathrm{~Hz}, 1 \mathrm{H})$, $2.39(\mathrm{~s}, 3 \mathrm{H}), 2.34 \sim 2.25(\mathrm{~m}, 2 \mathrm{H}), 1.66(\mathrm{~s}, 6 \mathrm{H}), 1.34(\mathrm{~s}$, $3 \mathrm{H}) ;{ }^{13} \mathrm{C}$ NMR $\left(100 \mathrm{MHz}, \mathrm{CDCl}_{3}\right) \delta: 208.9,199.8,149.7$, $147.4,142.6,137.4,136.3,136.1,136.0,126.1,124.0$, $112.5,77.3,77.0,76.7,55.7,51.8,43.2,38.3,29.0,25.3$, 21.1, 20.9, 16.0; IR (film) $v_{\max }: 2927,1708,1667,1568$, 1279, 1198, 987, 891, $778 \mathrm{~cm}^{-1}$; HRMS (ESI) calcd for $\mathrm{C}_{21} \mathrm{H}_{25} \mathrm{O}_{2}[\mathrm{M}+\mathrm{H}]^{+}$309.1849, found 309.1851.

( $R$ )-2-甲基-6-甲氧基-2-( $(1 R, 6 R)$-3-甲基-2-氧-6-异丙 烯基)-3,4-二氢狮- $1(2 H)$-酮(23): 从 2-甲基-6-甲氧基-1狮满酮 $(100 \mathrm{mg}$ )出发, 以 $65 \%$ 产率得到 $129 \mathrm{mg}$ 无色油状 液体 $\left[R_{\mathrm{f}}=0.38, V(\right.$ 正己烷 $): V($ 乙酸乙酯 $\left.)=8: 1\right] .[\alpha]_{\mathrm{D}}^{20}$ +75 (c 0.1, $\mathrm{CHCl}_{3}$ ); ${ }^{1} \mathrm{H}$ NMR (400 MHz, $\left.\mathrm{CDCl}_{3}\right) \delta: 7.65$ (d, $J=8.5 \mathrm{~Hz}, 1 \mathrm{H}), 6.90(\mathrm{dd}, J=8.5,2.2 \mathrm{~Hz}, 1 \mathrm{H}), 6.83(\mathrm{~d}$, $J=1.9 \mathrm{~Hz}, 1 \mathrm{H}), 6.56(\mathrm{~d}, J=2.7 \mathrm{~Hz}, 1 \mathrm{H}), 4.63 \sim 4.58(\mathrm{~m}$, $1 \mathrm{H}), 4.52(\mathrm{~s}, 1 \mathrm{H}), 3.88(\mathrm{~s}, 3 \mathrm{H}), 3.38(\mathrm{~d}, J=17.7 \mathrm{~Hz}, 1 \mathrm{H})$, $2.87(\mathrm{~d}, J=3.8 \mathrm{~Hz}, 1 \mathrm{H}), 2.78(\mathrm{~d}, J=17.7 \mathrm{~Hz}, 1 \mathrm{H}), 2.76 \sim$ $2.69(\mathrm{~m}, 1 \mathrm{H}), 2.46 \sim 2.18(\mathrm{~m}, 2 \mathrm{H}), 1.67(\mathrm{~s}, 6 \mathrm{H}), 1.35(\mathrm{~s}$, $3 \mathrm{H}) ;{ }^{13} \mathrm{C}$ NMR $\left(100 \mathrm{MHz}, \mathrm{CDCl}_{3}\right) \delta: 206.9,199.8,165.4$, $155.3,147.5,142.6,136.3,129.0,125.8,115.6,112.2$, 109.4, 77.3, 77.0, 76.7, 55.7, 55.6, 51.7, 42.9, 38.8, 28.8, 25.3, 21.0, $16.1 \mathrm{~cm}^{-1}$; IR (film) $v_{\max }: 2933,1823,1721$, $1558,1255,1125,962,878,782$; HRMS (ESI) calcd for $\mathrm{C}_{21} \mathrm{H}_{25} \mathrm{O}_{3}[\mathrm{M}+\mathrm{H}]^{+}$325.1798, found 325.1800.

( R)-2-甲基-6-氟-2-( $(1 R, 6 R)$-3-甲基-2-氧-6-异丙烯 基)-3,4-二氢狮-1(2H)-酮(24): 从 2-甲基-6-氟-1-狮满酮 (92 mg)出发, 以 54\%产率得到 $94.4 \mathrm{mg}$ 无色油状液体 $\left[R_{\mathrm{f}}=0.35, V(\right.$ 正己烷 $): V($ 乙酸乙酯 $\left.)=8: 1\right] .[\alpha]_{\mathrm{D}}^{20}+75$ (c $\left.0.15, \mathrm{CHCl}_{3}\right) ;{ }^{1} \mathrm{H}$ NMR $\left(400 \mathrm{MHz}, \mathrm{CDCl}_{3}\right) \delta: 7.39 \sim$ $7.27(\mathrm{~m}, 3 \mathrm{H}), 6.56(\mathrm{td}, J=4.0,1.3 \mathrm{~Hz}, 1 \mathrm{H}), 4.57 \sim 4.51(\mathrm{~m}$, $1 \mathrm{H}), 4.47$ (s, 1H), 3.36 (d, $J=17.4 \mathrm{~Hz}, 1 \mathrm{H}), 2.94$ (d, $J=$ $5.8 \mathrm{~Hz}, 1 \mathrm{H}), 2.88(\mathrm{~d}, J=17.4 \mathrm{~Hz}, 1 \mathrm{H}), 2.79$ (q, $J=5.6 \mathrm{~Hz}$, $1 \mathrm{H}), 2.28 \sim 2.37(\mathrm{~m}, 2 \mathrm{H}), 1.69 \sim 1.63(\mathrm{~m}, 6 \mathrm{H}), 1.37(\mathrm{~s}$, $3 \mathrm{H}) ;{ }^{19} \mathrm{~F}$ NMR $\left(377 \mathrm{MHz}, \mathrm{CDCl}_{3}\right) \delta:-114.46(\mathrm{~s}) ;{ }^{13} \mathrm{C}$ NMR $\left(100 \mathrm{MHz}, \mathrm{CDCl}_{3}\right) \delta: 207.8,199.9,162.3\left(\mathrm{~d},{ }^{1} J_{\mathrm{CF}}=\right.$ $246.2 \mathrm{~Hz}, \mathrm{C}-\mathrm{F}), 147.4\left(\mathrm{~d},{ }^{4} J_{\mathrm{CF}}=2.0 \mathrm{~Hz}\right), 147.0,142.5$, $137.8\left(\mathrm{~d}, \quad{ }^{3} J_{\mathrm{CF}}=7.1 \mathrm{~Hz}\right), 136.1,127.8\left(\mathrm{~d},{ }^{3} J_{\mathrm{CF}}=7.7 \mathrm{~Hz}\right.$, 
$\mathrm{CH}), 122.3\left(\mathrm{~d},{ }^{2} J_{\mathrm{CF}}=23.6 \mathrm{~Hz}, \mathrm{CH}\right), 113.4,109.7\left(\mathrm{~d},{ }^{2} J_{\mathrm{CF}}=\right.$ $21.5 \mathrm{~Hz}, \mathrm{CH}), 77.3,77.0,76.7,56.0,52.4,44.1,38.3,29.7$, 29.5, 25.2, 20.5, 16.0; IR (film) $v_{\text {max }}: 2922,1712,1662$, 1489, 1265, 1191, 932, 878, $732 \mathrm{~cm}^{-1}$; HRMS (ESI) calcd for $\mathrm{C}_{20} \mathrm{H}_{22} \mathrm{FO}_{2}[\mathrm{M}+\mathrm{H}]^{+}$313.1598, found 313.1595.

( $R$ )-2-甲基-5-甲氧基-2-( $(1 R, 6 R)$-3-甲基-2-氧-6-异丙 烯基)-3,4-二氢荎-1(2H)-酮(25)：从 2-甲基-5-甲氧基-1䒬酮(100 mg)出发, 以 64\%产率得到 $115 \mathrm{mg}$ 无色油状液 体 $25\left[\mathrm{R}_{\mathrm{f}}=0.37, V\right.$ (正己烷)： $V$ (乙酸乙酯 $\left.)=8: 1\right] .[\alpha]_{\mathrm{D}}^{20}$ +126 (c 0.4, $\left.\mathrm{CHCl}_{3}\right) ;{ }^{1} \mathrm{H}$ NMR (400 MHz, $\left.\mathrm{CDCl}_{3}\right) \delta: 7.66$ (dd, $J=7.9,0.6 \mathrm{~Hz}, 1 \mathrm{H}), 7.30 \sim 7.23(\mathrm{~m}, 1 \mathrm{H}), 6.99$ (d, $J=$ $7.5 \mathrm{~Hz}, 1 \mathrm{H}), 6.60$ (s, 1H), 4.68 (s, 2H), 3.85 (s, 3H), 3.01 (d, $J=2.1 \mathrm{~Hz}, 1 \mathrm{H}), 2.91$ (d, $J=7.1 \mathrm{~Hz}, 1 \mathrm{H}), 2.81$ (ddd, $J=$ 25.3, 13.3, $9.1 \mathrm{~Hz}, 2 \mathrm{H}), 2.74 \sim 2.62(\mathrm{~m}, 1 \mathrm{H}), 2.36 \sim 2.41$ $(\mathrm{m}, 1 \mathrm{H}), 2.35 \sim 2.26(\mathrm{~m}, 1 \mathrm{H}), 2.01 \sim 1.90(\mathrm{~m}, 1 \mathrm{H}), 1.77(\mathrm{~d}$, $J=1.6 \mathrm{~Hz}, 3 \mathrm{H}), 1.70$ (s, 3H), $1.23(\mathrm{~s}, 3 \mathrm{H}) ;{ }^{13} \mathrm{C}$ NMR $(100$ $\left.\mathrm{MHz}, \mathrm{CDCl}_{3}\right) \delta: 200.9,199.8,156.4,149.1,142.5,136.7$, 133.3, 130.9, 127.2, 120.0 113.6, 111.4, 77.3, 77.0, 76.7, 55.6, 52.8, 48.9, 41.4, 31.6, 29.7, 21.1, 20.5, 19.1, 16.2; IR (film) $v_{\max }: 2925,1745,1665,1548,1225,1195,954,878$, $784 \mathrm{~cm}^{-1}$; HRMS (ESI) calcd for $\mathrm{C}_{22} \mathrm{H}_{27} \mathrm{O}_{3}[\mathrm{M}+\mathrm{H}]^{+}$ 339.1955 , found 339.1955 .

( $R$ )-2-甲基-6-甲氧基-2-( $(1 R, 6 R)$-3-甲基-2-氧-6-异丙 烯基)-3,4-二氢萗- $1(2 H)$-酮(26): 从 2-甲基-6-甲氧基-1菜酮(100 mg)出发, 以 59\%产率得到 $105 \mathrm{mg}$ 无色油状液 体 $\left[R_{\mathrm{f}}=0.37, V(\right.$ 正己烷 $): V$ (乙酸乙酯 $\left.)=8: 1\right] .[\alpha]_{\mathrm{D}}^{20}+$ 107 (c $\left.0.2, \mathrm{CHCl}_{3}\right) ;{ }^{1} \mathrm{H}$ NMR (400 MHz, $\left.\mathrm{CDCl}_{3}\right) \delta: 8.00$ (dd, $J=8.8,1.3 \mathrm{~Hz}, 1 \mathrm{H}), 6.83(\mathrm{~d}, J=8.8 \mathrm{~Hz}, 1 \mathrm{H}), 6.63(\mathrm{~d}$, $J=1.6 \mathrm{~Hz}, 1 \mathrm{H}), 6.58(\mathrm{~s}, 1 \mathrm{H}), 4.65(\mathrm{dd}, J=3.4,1.9 \mathrm{~Hz}$, $2 \mathrm{H}), 3.82(\mathrm{~d}, J=1.3 \mathrm{~Hz}, 3 \mathrm{H}), 3.05$ (s, 1H), 2.91 (dd, $J=$ 9.1, $4.9 \mathrm{~Hz}, 1 \mathrm{H}), 2.86 \sim 2.79(\mathrm{~m}, 2 \mathrm{H}), 2.71 \sim 2.57(\mathrm{~m}, 1 \mathrm{H})$, $2.36 \sim 2.43(\mathrm{~m}, 1 \mathrm{H}), 2.35 \sim 2.23(\mathrm{~m}, 1 \mathrm{H}), 1.91(\mathrm{dt}, J=$ 11.4, $5.2 \mathrm{~Hz}, 1 \mathrm{H}), 1.76$ (s, 3H), 1.70 (s, 3H), 1.23 (d, $J=$ $1.1 \mathrm{~Hz}, 3 \mathrm{H}) ;{ }^{13} \mathrm{C}$ NMR $\left(100 \mathrm{MHz}, \mathrm{CDCl}_{3}\right) \delta: 200.2,199.1$, $163.3,149.1,144.5,142.5,136.7,130.7,125.7,113.5$, 112.2, 111.1, 77.4, 77.0, 76.7, 55.4, 53.6, 48.9, 41.7, 31.7, 29.7, 25.6, 21.3, 21.1, 16.3; IR (film) $v_{\max }$ : 2923, 1741, 1670, 1548, 1223, 1159, 944, 858, $789 \mathrm{~cm}^{-1}$; HRMS (ESI) calcd for $\mathrm{C}_{22} \mathrm{H}_{27} \mathrm{O}_{3}[\mathrm{M}+\mathrm{H}]^{+}$339.1955, found 339.1952.

( $R$ )-2-甲基-7-甲氧基-2-(( $1 R, 6 R)$-3-甲基-2-氧-6-异丙 烯基)-3,4-二氢萗-1(2H)-酮(27): 从 2-甲基-7-甲氧基-1萗酮(100 mg)出发, 以 65\%产率得到 $116 \mathrm{mg}$ 无色油状液 体 $\left[R_{\mathrm{f}}=0.37, V\right.$ (正己烷) $: V$ (乙酸乙酯) $\left.=8: 1\right] .[\alpha]_{\mathrm{D}}^{20}+$ $70\left(c 0.1, \mathrm{CHCl}_{3}\right) ;{ }^{1} \mathrm{H}$ NMR $\left(400 \mathrm{MHz}, \mathrm{CDCl}_{3}\right) \delta: 7.54(\mathrm{~d}$, $J=2.8 \mathrm{~Hz}, 1 \mathrm{H}), 7.11(\mathrm{~d}, J=8.4 \mathrm{~Hz}, 1 \mathrm{H}), 7.04$ (dd, $J=8.4$,
$2.8 \mathrm{~Hz}, 1 \mathrm{H}), 6.62(\mathrm{~s}, 1 \mathrm{H}), 4.72 \sim 4.63(\mathrm{~m}, 2 \mathrm{H}), 3.85(\mathrm{~s}$, $3 \mathrm{H}), 3.06(\mathrm{~d}, J=2.2 \mathrm{~Hz}, 1 \mathrm{H}), 2.87(\mathrm{dd}, J=12.4,7.3 \mathrm{~Hz}$, $3 \mathrm{H}), 2.63 \sim 2.71(\mathrm{~m}, 1 \mathrm{H}), 2.38 \sim 2.45(\mathrm{~m}, 1 \mathrm{H}), 2.38 \sim 2.27$ $(\mathrm{m}, 1 \mathrm{H}), 2.01 \sim 1.91(\mathrm{~m}, 1 \mathrm{H}), 1.79(\mathrm{~d}, J=1.5 \mathrm{~Hz}, 3 \mathrm{H})$, $1.72(\mathrm{~s}, 3 \mathrm{H}), 1.25(\mathrm{~s}, 3 \mathrm{H}) ;{ }^{13} \mathrm{C}$ NMR $\left(100 \mathrm{MHz}, \mathrm{CDCl}_{3}\right) \delta$ : $200.5,200.0,158.5,149.1,142.5,136.7,134.5,133.1$, 129.7, 121.6, 111.4, 110.0, 77.3, 77.0, 76.7, 55.5, 53.2, 49.1, 41.7, 32.2, 29.8, 24.4, 21.1, 20.8, 16.3; IR (film) $v_{\max }$ : 2923, 1748, 1689, 1568, 1242, 1189, 938, 887, $748 \mathrm{~cm}^{-1}$; HRMS (ESI) calcd for $\mathrm{C}_{22} \mathrm{H}_{27} \mathrm{O}_{3}[\mathrm{M}+\mathrm{H}]^{+} 339.1955$, found 339.1955 .

(R)-2-甲基-7-澳-2-((1R,6R)-3-甲基-2-氧-6-异丙烯

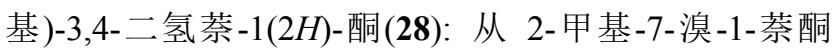
$(100 \mathrm{mg})$ 出发, 以 $55 \%$ 产率得到 $89.1 \mathrm{mg}$ 无色油状液体 $\left[R_{\mathrm{f}}=0.35, V\right.$ (正己烷 $): V$ (乙酸乙酯 $\left.)=8: 1\right] .[\alpha]_{\mathrm{D}}^{20}+45$ (c 0.1, $\left.\mathrm{CHCl}_{3}\right) ;{ }^{1} \mathrm{H}$ NMR (400 MHz, $\left.\mathrm{CDCl}_{3}\right) \delta: 8.16$ (d, $J=$ $2.2 \mathrm{~Hz}, 1 \mathrm{H}), 7.55$ (dd, $J=8.2,2.2 \mathrm{~Hz}, 1 \mathrm{H}), 7.08$ (d, $J=8.2$ $\mathrm{Hz}, 1 \mathrm{H}), 6.62$ (s, 1H), 4.68 (d, $J=1.4 \mathrm{~Hz}, 2 \mathrm{H}), 3.00$ (d, $J=$ $2.8 \mathrm{~Hz}, 1 \mathrm{H}), 2.95 \sim 2.79(\mathrm{~m}, 3 \mathrm{H}), 2.71 \sim 2.58(\mathrm{~m}, 1 \mathrm{H})$, $2.47 \sim 2.39(\mathrm{~m}, 1 \mathrm{H}), 2.31 \sim 2.38(\mathrm{~m}, 1 \mathrm{H}), 2.01 \sim 1.90(\mathrm{~m}$, $1 \mathrm{H}), 1.77$ (d, $J=1.5 \mathrm{~Hz}, 3 \mathrm{H}), 1.70$ (s, 3H), 1.25 (s, 3H); ${ }^{13} \mathrm{C}$ NMR (100 MHz, $\left.\mathrm{CDCl}_{3}\right) \delta: 199.6,199.4,148.8,142.7$, $140.5,136.6,135.6,134.0,131.1,130.2,120.9,111.8$, 77.3, 77.0, 76.7, 53.1, 49.0, 41.8, 31.9, 29.8, 24.7, 20.9, 20.6, 16.2; IR (film) $v_{\max }: 2925,1735,1680,1578,1232$, 1228, 935, 878, $775 \mathrm{~cm}^{-1}$; HRMS (ESI) calcd for $\mathrm{C}_{21} \mathrm{H}_{24} \mathrm{BrO}_{2}[\mathrm{M}+\mathrm{H}]^{+}$387.0954, found 387.0957.

( $R$ )-2-甲基-5-苯乙酰氧基-2-( $(1 R, 6 R)$-3-甲基-2-氧-6异丙烯基)-3,4-二氢荎-1(2H)-酮(29)：从 2-甲基-5-苯乙 酰氧基-1-䒬酮 $(100 \mathrm{mg}$ )出发, 以 $60 \%$ 产率得到 $89.9 \mathrm{mg}$ 无色油状液体 $\left[R_{\mathrm{f}}=0.33, V(\right.$ 正己烷 $): V($ 乙酸乙酯 $)=8$ : 1]. $[\alpha]_{\mathrm{D}}^{20}+55\left(\right.$ c $\left.0.1, \mathrm{CHCl}_{3}\right) ;{ }^{1} \mathrm{H} \mathrm{NMR}\left(400 \mathrm{MHz}, \mathrm{CDCl}_{3}\right)$ $\delta: 8.27 \sim 8.16(\mathrm{~m}, 2 \mathrm{H}), 8.03(\mathrm{dd}, J=7.7,1.4 \mathrm{~Hz}, 1 \mathrm{H}), 7.67$ (dd, $J=10.6,4.3 \mathrm{~Hz}, 1 \mathrm{H}), 7.54(\mathrm{t}, J=7.7 \mathrm{~Hz}, 2 \mathrm{H}), 7.42$ (t, $J=7.8 \mathrm{~Hz}, 1 \mathrm{H}), 7.37(\mathrm{dd}, J=7.9,1.4 \mathrm{~Hz}, 1 \mathrm{H}), 6.61(\mathrm{~s}$, $1 \mathrm{H}), 4.70(\mathrm{dd}, J=4.6,3.3 \mathrm{~Hz}, 2 \mathrm{H}), 3.07$ (d, $J=2.6 \mathrm{~Hz}$, $1 \mathrm{H}), 2.91 \sim 2.85(\mathrm{~m}, 1 \mathrm{H}), 2.82(\mathrm{dd}, J=13.0,7.1 \mathrm{~Hz}, 2 \mathrm{H})$, $2.70 \sim 2.60(\mathrm{~m}, 1 \mathrm{H}), 2.47 \sim 2.39(\mathrm{~m}, 1 \mathrm{H}), 2.39 \sim 2.29(\mathrm{~m}$, 1H), 1.97 (dt, $J=13.8,5.9 \mathrm{~Hz}, 1 \mathrm{H}), 1.77(\mathrm{~d}, J=1.3 \mathrm{~Hz}$, 3H), 1.73 (s, 3H), $1.26(\mathrm{~s}, 3 \mathrm{H}) ;{ }^{13} \mathrm{C}$ NMR $(100 \mathrm{MHz}$, $\left.\mathrm{CDCl}_{3}\right) \delta: 199.7,199.7,164.5,148.8,148.3,142.6,136.7$, $134.3,133.9,133.9,130.2,128.7,127.4,126.5,126.2$, 111.6, 77.3, 77.0, 76.7, 53.2, 48.8, 41.8, 31.0, 29.8, 21.0, 20.8, 19.6, 16.2; IR (film) $v_{\text {max }}: 2922,1740,1660,1568$, $1230,1089,935,867,712 \mathrm{~cm}^{-1}$; HRMS (ESI) calcd for 
$\mathrm{C}_{28} \mathrm{H}_{29} \mathrm{O}_{4}[\mathrm{M}+\mathrm{H}]^{+}$429.2060, found 429.2064.

(R)-2- 甲基 -2-(( $1 R, 6 R)$-3- 甲基 -2- 氧 -6- 异丙烯 基)-3,4-二氢苯并呋喃-1(2H)-酩(30): 从 2-甲基-1-二氢 苯并呋喃酮 $(100 \mathrm{mg})$ 出发, 以 $62 \%$ 得到 $123 \mathrm{mg}$ 白色固体 $\left[R_{\mathrm{f}}=0.38, V\right.$ (正己烷) $: V$ (乙酸乙酯 $\left.)=8 ： 1\right]$. m.p. 159 $163{ }^{\circ} \mathrm{C} ;[\alpha]_{\mathrm{D}}^{20}+105\left(c 0.35, \mathrm{CHCl}_{3}\right) ;{ }^{1} \mathrm{H}$ NMR $(400 \mathrm{MHz}$, $\left.\mathrm{CDCl}_{3}\right) \delta: 7.33(\mathrm{~d}, J=2.0 \mathrm{~Hz}, 1 \mathrm{H}), 6.69(\mathrm{~d}, J=2.0 \mathrm{~Hz}$, $1 \mathrm{H}), 6.59$ (d, J=0.9 Hz, 1H), $4.74 \sim 4.69$ (m, 1H), 4.68 (s, $1 \mathrm{H}), 2.98(\mathrm{~d}, J=2.4 \mathrm{~Hz}, 1 \mathrm{H}), 2.90 \sim 2.82(\mathrm{~m}, 3 \mathrm{H}), 2.74 \sim$ $2.60(\mathrm{~m}, 1 \mathrm{H}), 2.45 \sim 2.52(\mathrm{~m}, 1 \mathrm{H}), 2.38 \sim 2.27(\mathrm{~m}, 1 \mathrm{H})$, $1.97(\mathrm{dt}, \quad J=13.6,5.5 \mathrm{~Hz}, 1 \mathrm{H}), 1.75(\mathrm{~d}, J=1.9 \mathrm{~Hz}, 3 \mathrm{H})$, $1.73(\mathrm{~d}, J=0.6 \mathrm{~Hz}, 3 \mathrm{H}), 1.24$ (s, 3H); ${ }^{13} \mathrm{C} \mathrm{NMR}(100 \mathrm{MHz}$, $\left.\mathrm{CDCl}_{3}\right) \delta: 200.3,196.8,164.1,149.0,143.0,142.5,136.6$, 120.2, 111.2, 107.3, 77.3, 77.0, 76.7, 54.0, 49.7, 42.1, 32.0, 29.7, 21.6, 21.1, 20.6, 16.3; IR (film) $v_{\max }: 2922,1743$, 1658, 1578, 1245, 1119, 956, 887, $738 \mathrm{~cm}^{-1}$; HRMS (ESI) calcd for $\mathrm{C}_{19} \mathrm{H}_{23} \mathrm{O}_{3}[\mathrm{M}+\mathrm{H}]^{+}$299.1642, found 299.1644.

(R)-3-甲基-3-((1R,6R)-3-甲基-2-氧-6-异丙烯基)-1色满酮(31): 从 2-甲基-4-色满酮(100 mg)出发, 以 57\% 产率得到 $110 \mathrm{mg}$ 无色油状液体 $\left[R_{\mathrm{f}}=0.36, V\right.$ (正己烷) : $V($ 乙酸乙酯 $)=8: 1] .[\alpha]_{\mathrm{D}}^{20}+160\left(c \quad 0.3, \mathrm{CHCl}_{3}\right) ;{ }^{1} \mathrm{H}$ NMR (400 MHz, $\left.\mathrm{CDCl}_{3}\right) \delta: 7.90(\mathrm{dd}, J=7.9,1.7 \mathrm{~Hz}, 1 \mathrm{H})$, $7.44(\mathrm{ddd}, J=8.7,7.2,1.7 \mathrm{~Hz}, 1 \mathrm{H}), 7.10 \sim 6.98(\mathrm{~m}, 1 \mathrm{H})$, $6.91(\mathrm{~d}, J=8.3 \mathrm{~Hz}, 1 \mathrm{H}), 6.48(\mathrm{~d}, J=3.2 \mathrm{~Hz}, 1 \mathrm{H}), 4.73$ (dd, $J=8.0,6.7 \mathrm{~Hz}, 2 \mathrm{H}), 4.64$ (d, $J=11.9 \mathrm{~Hz}, 1 \mathrm{H}), 4.11$ (d, $J=$ $11.9 \mathrm{~Hz}, 1 \mathrm{H}), 2.99$ (dt, $J=6.5,3.1 \mathrm{~Hz}, 1 \mathrm{H}), 2.87$ (d, $J=3.3$ $\mathrm{Hz}, 1 \mathrm{H}), 2.48 \sim 2.55(\mathrm{~m}, 1 \mathrm{H}), 2.29 \sim 2.36(\mathrm{~m}, 1 \mathrm{H}), 1.74(\mathrm{~s}$, $3 \mathrm{H}), 1.69$ (d, $J=1.5 \mathrm{~Hz}, 3 \mathrm{H}), 1.24(\mathrm{~s}, 3 \mathrm{H}) ;{ }^{13} \mathrm{C}$ NMR $(100$ $\left.\mathrm{MHz}, \mathrm{CDCl}_{3}\right) \delta: 198.9,195.8,160.5,148.0,142.7,136.2$, $135.4,127.8,121.8,120.7,117.3,111.7,77.3,77.0,76.7$, 73.7, 52.6, 48.4, 41.5, 29.4, 21.0, 17.5, 16.1; IR (film) $v_{\max }$ : 2923, 1742, 1668, 1482, 1218, 1173, 925, 889, $732 \mathrm{~cm}^{-1}$; HRMS (ESI) calcd for $\mathrm{C}_{20} \mathrm{H}_{23} \mathrm{O}_{3}[\mathrm{M}+\mathrm{H}]^{+}$311.1642, found 311.1640 .

\subsection{3 对照实验}

将四氢荎酮底物 $10(0.1 \mathrm{~mL}, 0.66 \mathrm{mmol})$ 和 $(R)$-香芹 酮 $13(0.1 \mathrm{~mL}, 0.66 \mathrm{mmol})$ 溶解在四氢呋喃 $(3.0 \mathrm{~mL})$ 中, 在 $-78{ }^{\circ} \mathrm{C}$ 和氩气氛围下逐滴加入二异丙基氨基锂 $(2$ $\mathrm{mol} \cdot \mathrm{L}^{-1}$, 溶于 THF, $\left.0.72 \mathrm{~mL}, 1.44 \mathrm{mmol}\right)$, 在此温度下 搅拌反应 $1 \mathrm{~h}$; 然后在 $-78{ }^{\circ} \mathrm{C}$ 和氩气氛围下逐滴加入溶 解在 $N, N$-二甲基甲酰胺(DMF) (3.0 mL)中的无水三氯化 铁(235 mg, $1.44 \mathrm{mmol})$, 之后缓慢升至室温并在此温度 下搅拌反应 $8 \mathrm{~h}$, 用饱和氯化铵水溶液淬灭反应, 然后 用乙醚萃取水相, 合并所得有机相并用无水硫酸钠干 燥. 减压浓缩后, 残余物经制备色谱分离 $[V$ (正己烷)：
$V($ 二氯乙烷 $)=1 ： 4]$, 以 $9 \%$ 的收率得到 $18.3 \mathrm{mg}$ 偶联产 物 11; 以 $19 \%$ 的收率得到 $38.6 \mathrm{mg}$ 的另一非对映异构体 32. m.p. $103 \sim 106{ }^{\circ} \mathrm{C}$; $[\alpha]_{\mathrm{D}}^{20}+5.6\left(c 0.25, \mathrm{CHCl}_{3}\right) ;{ }^{1} \mathrm{H}$ NMR (500 MHz, $\left.\mathrm{CDCl}_{3}\right) \delta: 8.07(\mathrm{~d}, J=7.8 \mathrm{~Hz}, 1 \mathrm{H}), 7.44$ $(\mathrm{td}, J=7.5,1.3 \mathrm{~Hz}, 1 \mathrm{H}), 7.32(\mathrm{t}, J=7.5 \mathrm{~Hz}, 1 \mathrm{H}), 7.21$ (d, $J=7.6 \mathrm{~Hz}, 1 \mathrm{H}), 6.66(\mathrm{ddd}, J=4.8,3.4,1.4 \mathrm{~Hz}, 1 \mathrm{H}), 4.82$ $(\mathrm{s}, 1 \mathrm{H}), 4.77 \sim 4.70(\mathrm{~m}, 1 \mathrm{H}), 3.40(\mathrm{~d}, J=9.7 \mathrm{~Hz}, 1 \mathrm{H}), 3.01$ (ddd, $J=15.1,13.6,8.6 \mathrm{~Hz}, 2 \mathrm{H}), 2.86 \sim 2.76(\mathrm{~m}, 1 \mathrm{H})$, $2.59 \sim 2.47(\mathrm{~m}, 1 \mathrm{H}), 237 \sim 2.42(\mathrm{~m}, 1 \mathrm{H}), 2.06 \sim 2.10(\mathrm{~m}$, $1 \mathrm{H}), 1.95(\mathrm{dt}, J=13.2,5.3 \mathrm{~Hz}, 1 \mathrm{H}), 1.72$ (d, $J=1.6 \mathrm{~Hz}$, $3 \mathrm{H}), 1.69(\mathrm{~s}, 3 \mathrm{H}), 1.25(\mathrm{~s}, 3 \mathrm{H}) ;{ }^{13} \mathrm{C} \mathrm{NMR}(125 \mathrm{MHz}$, $\left.\mathrm{CDCl}_{3}\right) \delta: 201.1,199.1,147.6,143.3,142.0,135.8,132.7$, $132.1,128.6,128.0,126.7,113.2,77.3,77.0,76.8,54.2$, 47.1, 45.0, 31.6, 30.1, 25.0, 19.9, 19.5, 16.1; IR (film) $v_{\max }$ : 2990, 1769, 1376, 1247, 1057, 748; HRMS (ESI) calcd for $\mathrm{C}_{19} \mathrm{H}_{32} \mathrm{NO}_{2}[\mathrm{M}+\mathrm{H}]^{+}$309.1849, found 309.1843.

\section{2 .4 自由基捕获实验}

将硝酸铈铵 (311 mg, $0.52 \mathrm{mmol}$ )、2,6-二叔丁基吡啶 (0.18 mL, $0.78 \mathrm{mmol}$ )、2,2,6,6-四甲基哌啶氧化物(88 mg, $0.52 \mathrm{mmol})$ 溶解在乙腈 $(4.0 \mathrm{~mL})$ 中, 然后在 $-30{ }^{\circ} \mathrm{C}$ 和氩 气氛围下通过导管加入双烯醇硅醚底物 14 (109 mg, 溶 解在 $1.0 \mathrm{~mL}$ 的四氢呋喃中), 在此温度下搅拌反应 15 $\min$ 后, 用饱和氯化铵水溶液 $(3.0 \mathrm{~mL})$ 淬灭反应, 用乙酸 乙酯 $(5.0 \mathrm{~mL} \times 3)$ 萃取水相, 合并所得有机相用无水硫酸 钠干燥. 减压浓缩后, 残余物经快速柱色谱分离 $[V($ 正己 烷 $): V($ 乙酸乙酯 $)=30: 1]$, 以 $65 \%$ 的收率得到 $51 \mathrm{mg}$ 针状晶体 $33\left[R_{\mathrm{f}}=0.35, V(\right.$ 正己烷 $): V($ 乙酸乙酯 $)=4$ : 1]. m.p. $66 \sim 69{ }^{\circ} \mathrm{C} ;[\alpha]_{\mathrm{D}}^{20}-60\left(c \quad 0.26, \mathrm{CHCl}_{3}\right) ;{ }^{1} \mathrm{H}$ NMR $\left(400 \mathrm{MHz}, \mathrm{CDCl}_{3}\right) \delta: 6.65 \sim 6.51(\mathrm{~m}, 1 \mathrm{H}), 4.82(\mathrm{~d}$, $J=1.2 \mathrm{~Hz}, 1 \mathrm{H}), 4.65$ (s, 1H), 4.15 (d, $J=2.6 \mathrm{~Hz}, 1 \mathrm{H}), 3.09$ (s, 1H), 2.87 (ddt, $J=19.2,5.5,2.7 \mathrm{~Hz}, 1 \mathrm{H}$ ), 2.31 (ddd, $J=19.1,5.6,1.0 \mathrm{~Hz}, 1 \mathrm{H}), 1.75$ (dt, $J=2.7,1.4 \mathrm{~Hz}, 3 \mathrm{H})$, 1.73 (s, 3H), 1.50 (d, $J=26.9 \mathrm{~Hz}, 3 \mathrm{H}), 1.43$ (d, $J=4.4 \mathrm{~Hz}$, $3 \mathrm{H}), 1.27$ (d, $J=12.0 \mathrm{~Hz}, 3 \mathrm{H}), 1.14$ (s, 3H), 1.02 (s, 3H), $0.94 \sim 0.82(\mathrm{~m}, 3 \mathrm{H}) ;{ }^{13} \mathrm{C}$ NMR $\left(100 \mathrm{MHz}, \mathrm{CDCl}_{3}\right) \delta:$ $198.3,142.1$ ( $\times 2), 134.0,112.7,85.6,77.4,77.0,76.8$, 46.6, 39.9, 26.7, 22.3, 17.2, 16.0; IR (film) $v_{\max }: 2923$, 1715, 1674, 1449, 1364, 1105, $773 \mathrm{~cm}^{-1}$; HRMS (ESI) calcd for $\mathrm{C}_{19} \mathrm{H}_{32} \mathrm{NO}_{2}[\mathrm{M}+\mathrm{H}]^{+}$306.2428, found 306.2427.

辅助材料(Supporting Information) 包含所有新化合 物的 ${ }^{1} \mathrm{H}$ NMR, ${ }^{13} \mathrm{C}$ NMR, DEPT135, 2D NMR 谱图以及 化合物 15,30 和 32 的 $X$ 射线晶体衍射图与数据. 这些 材料可以免费从本刊网站(http://sioc-journal.cn/)上下载. 


\section{References}

[1] For selected reviews, see: (a) Csákÿ, A. G.; Plumet, J. Chem. Soc. Rev. 2001, 30, 313.

(b) Yeung, C. S.; Dong, V. M. Chem. Rev. 2011, 111, 1215.

(c) Guo, F. H.; Clift, M. D.; Thomson, R. J. Eur. J. Org. Chem. 2012, 4881 .

(d) Murarka, S.; Antonchick, A. P. Synthesis 2018, 50, 2150.

[2] Ivanoff, D.; Spassoff, A. Bull. Soc. Chim. Fr. 1935, 2, 76.

[3] For selected oxidative coupling examples, see: (a) Rathke, M. W.; Lindert, A. J. Am. Chem. Soc. 1971, 93, 4605.

(b) Ito, Y.; Konoike, T.; Saegusa, T. J. Am. Chem. Soc. 1975, 97, 649.

(c) Ito, Y.; Konoike, T.; Harada, T.; Saegusa, T. J. Am. Chem. Soc. 1977, 99, 1487.

(d) Frazier, Jr. R.; Harlow, R. J. Org. Chem. 1980, 45, 5408.

(e) Moeller, K. D.; Tinao, L. V. J. Am. Chem. Soc. 1992, 114, 1033.

(f) Mazzega, M.; Fabris, F.; Cossu, S.; Lucchi, O. D.; Lucchini, V.; Valle, G. Tetrahedron 1999, 55, 4427.

(g) Jang, H. Y.; Hong, J. B.; MacMillan, D. W. C. J. Am. Chem. Soc. 2007, 129, 7004.

(h) DeMartino, M. P.; Chen, K.; Baran, P. S. J. Am. Chem. Soc. 2008, 130, 11546 .

(i) Casey, B. M.; Flowers, R. A. J. Am. Chem. Soc. 2011, 133, 11492.

(j) Amaya, T.; Maegawa, Y.; Masuda, T.; Osafune, Y.; Hirao. T. J. Am. Chem. Soc. 2015, 137, 10072.

(k) Kaiser, D.; Teskey, C. J.; Alder, P.; Maulide. N. J. Am. Chem. Soc. 2017, 139, 16040.

(1) Næsborg, L.; Corti, V.; Leth, L. A.; Poulsen, P. H.; Jørgensen. A. Angew. Chem., Int. Ed. 2018, 57, 1606.

(m) Tanaka, T.; Tanaka, T.; Tsuji, T.; Yazaki, R.; Ohshima, T. Org. Lett. 2018, 20, 3541.

[4] For selected applications of oxidative coupling reaction in natural product synthesis, see: (a) Baran, P. S.; Guerrero, C. A.; Ambhaikar, N. B.; Hafensteiner, B. D. Angew. Chem., Int. Ed. 2005, 44, 606.

(b) Baran, P. S.; Hafensteiner, B. D.; Ambhaikar, N. B.; Guerrero, C. A.; Gallagher, J. D. J. Am. Chem. Soc. 2006, 128, 8678.

(c) Martin, C. L.; Overman, L. E.; Rohde, J. M. J. Am. Chem. Soc. 2008, 130, 7568 .

(d) Herzon, S. B.; Lu, L.; Woo, C. M.; Gholap, S. L. J. Am. Chem. Soc. 2011, 133, 7260 .

(e) Konkol, L. C.; Guo, F. H.; Sarjeant, A. A.; Thomson, R. J. Angew. Chem., Int. Ed. 2011, 50, 9931.

(f) Jones, B. T.; Avetta, C. T.; Thomson, R. J. Chem. Sci. 2014, 5, 1794.

(g) You, L.; Liang, X.-T.; Xu, L.-M.; Wang, Y.-F.; Zhang, J.-J.; Su, Q.; Li, Y.-H.; Zhang, B.; Yang, S.-L.; Chen, J.-H.; Yang, Z. J. Am. Chem. Soc. 2015, 137, 10120.

(h) Robison, E. E.; Thomson, R. J. J. Am. Chem. Soc. 2018, 140, 1956.

[5] Wender, P. A.; Miller, B. L. In Organic Synthesis: Theory and Applications, Vol. 2, Ed.: Hudlicky, T., JAI Press, Greenwich, CT, 1993.

[6] (a) Tokuda, M.; Shigei, T.; Itoh, M. Chem. Lett. 1975, 621.

(b) Baciocchi, E.; Casu, A.; Ruzziconi, R. Tetrahedron Lett. 1989, 30, 3707.

[7] (a) Beeson, T. D.; Mastracchio, A.; Hong, J. B.; Ashton, K.; MacMillan, D. W. C. Science 2007, 316, 582.

(b) Yasu, Y.; Koike, T.; Akita, M. Chem. Commun. 2012, 48, 5355.

[8] (a) Schmittel, M.; Burghart, A.; Malisch, W.; Reising, J.; Söllner, R. J. Org. Chem. 1998, 63, 396.

(b) Schmittel, M.; Haeuseler, A. J. Organomet. Chem. 2002, 661, 169.
[9] (a) Avetta, C. T.; Konkol, L. C.; Taylor, C. N.; Dugan, K. C.; Stern, C. L.; Thomson, R. J. Org. Lett. 2008, 10, 5621.

(b) Konkol, L. C.; Jones, B. T.; Thomson, R. J. Org. Lett. 2009, 11, 5550 .

[10] (a) Fuji, K. Chem. Rev. 1993, 93, 2037.

(b) Corey, E. J.; Guzman-Perez, A. Angew. Chem., Int. Ed. 1998 , 37, 388

(c) Christoffers, J.; Mann, A. Angew. Chem., Int. Ed. 2001, 40, 4591.

(d) Douglas, C. J.; Overman, L. E. Proc. Natl. Acad. Sci. U. S. A. 2004, 101, 5363 .

(e) Christoffers, J.; Baro, A. Quaternary Stereocenters: Challenges and Solutions for Organic Synthesis, Wiley-VCH, Weinheim, 2005. (f) Trost, B. M.; Jiang, C. Synthesis 2006, 369.

(g) Hawner. C.; Alexakis, A. Chem. Commun. 2010, 46, 7295.

(h) Shimizu, M. Angew. Chem., Int. Ed. 2011, 50, 5998.

(i) Hong, A. Y.; Stoltz, B. M. Eur. J. Org. Chem. 2013, 2745.

(j) Quasdorf, K. W.; Overman, L. E. Nature 2014, 516, 181.

(k) Long, R.; Huang, J.; Gong, J. X.; Yang, Z. Nat. Prod. Rep. 2015, $32,1584$.

[11] (a) Arya, P.; Joseph, R.; Chou, D. T. H. Chem. Biol. 2002, 9, 145. (b) Juncosa Jr, J. I.; Hansen, M.; Bonner, L. A.; Cueva, J. P.; Maglathlin, R.; McCorvy, J. D.; Marona-Lewicka, D.; Lill, M. A.; Nichols, D. E. ACS Chem. Neurosci. 2013, 4, 96.

[12] (a) Narayanan, C. R.; Pachapurkar, R. V. Tetrahedron Lett. 1965, 48,4333 .

(b) Ziffer, H.; Weiss, U.; Narayanan, C. R.; Pachapurkar, R. V. J. Org. Chem. 1966, 31, 2691.

(c) Harris, M.; Henderson, R.; McCrindle, R.; Overton, K. H.; Turner, D. W. Tetrahedron 1968, 24, 1517.

[13] Fontana, A.; Muniain, C.; Cimino, G. J. Nat. Prod. 1998, 61, 1027.

[14] Gonzalez, M. Curr. Bioact. Compd. 2007, 3, 1.

[15] (a) Zhang, P. P.; Yan, Z. M.; Li, Y. H.; Gong, G. X.; Yang, Z. J. Am. Chem. Soc. 2017, 139, 13989.

(b) Huang, J.; Gu, Y. Q.; Guo, K.; Zhu, L.; Lan, Y.; Gong, G. X.; Yang, Z. Angew. Chem., Int. Ed. 2017, 56, 7890.

(c) Liu, D.-D.; Sun, T.-W.; Wang, K.-Y.; Lu, Y.; Zhang, S.-L.; Li, Y.-H.; Jiang, Y.-L.; Chen, J.-H.; Yang, Z. J. Am. Chem. Soc. 2017, 139, 5732.

(d) Huang, Z. H.; Huang, J.; Qu, Y. Z.; Zhang, W. B.; Gong, J. X.; Yang, Z. Angew. Chem., Int. Ed. 2018, 57, 8744.

[16] Paquette, L. A.; Bzowej, E. I.; Branan, B. M.; Stanton, K. J. J. Org. Chem. 1995, 60, 7277.

[17] (a) Dessau, R. M.; Heiba, E. I. J. Org. Chem. 1974, 39, 3457. (b) Liu, X. G.; Chen, X. H.; Mohr, J. T. Org. Lett. 2016, 18, 3182.

[18] Kobayashi, Y.; Taguchi, T.; Tokuno, E. Tetrahedron Lett. 1977, 3741.

[19] CDCC 1501822 (15), CDCC 1582641 (30) and CDCC 1861685 (32) contains the supplementary crystallographic data for this paper. These data can be obtained free of charge via www.ccdc.cam.ac. uk/data request/cif, by emailing data request@ccdc.cam.ac.uk, or by contacting The Cambridge Crystallographic Data Centre, 12 Union Road, Cambridge CB2 1EZ, U. K.; fax: +44-1223 336033.

[20] (a) Chatgilialoglu, C.; Studer, A. Encyclopedia of Radicals in Chemistry, Biology and Materials, Vols. 1 4, Wiley, Chichester, 2012.

(b) Zhang, N.; Samanta, S. R.; Rosen, B. M.; Percec, V. Chem. Rev. 2014, 114, 5848 .

(c) Studer, A.; Curran, D. P. Angew. Chem., Int. Ed. 2016, 55, 58.

(d) Zard, S. Z. Org. Lett. 2017, 19, 1257.

[21] Frazier, Jr. R.; Harlow, R. J. Org. Chem. 1980, 45, 5408.

[22] (a) Schmittel, M.; Söllner, R. Chem. Ber./Rec. 1997, 130, 771. (b) Schmittel, M.; Burghart, A.; Werner, H.; Laubender, M.; Söllner, R. J. Org. Chem. 1999, 104, 3077. 